\title{
Sustainable and renewable implementation multi-criteria energy model (SRIME)—case study: Sri Lanka
}

\author{
Luis Carlos Domínguez-Dafauce • \\ Francisco Marcos Martín
}

Received: 3 September 2014 / Accepted: 4 February 2015/Published online: 20 February 2015

(C) The Author(s) 2015. This article is published with open access at Springerlink.com

\begin{abstract}
Sustainable and renewable are certainly very appreciated terms nowadays. These words may summarize a whole new attitude towards our world and the people who live in it. This paper's goal is to define an original multicriteria energy model, named SRIME, specially designed for developing countries. First, an extensive research will be carried out on: energy demand; potential renewable energy, its current know-how and potential future development; potential avoided emissions $\left(\mathrm{CO}_{2}, \mathrm{NO}_{\mathrm{X}}, \mathrm{SO}_{2}\right)$; and the possible international support versus the in-country possibilities. The precedence constraints will be applied to establish in which degree renewable energy may be substituting for the fossil fuel: the purely economic approach will give way to a sustainable, renewable, development focused energy planning. It must be noted that an innovative function has been specifically included in the SRIME, which evaluates, applying the precedence constraints, the influence renewable energy may have on developing countries rural health and education. Six functions have been established: replaceable amount of fossil energy; $\mathrm{CO}_{2}, \mathrm{NO}_{\mathrm{X}}$ and $\mathrm{SO}_{2}$ avoidable emissions; rural health and education development maximization; and the cost function. These functions will be optimized through the Chebyshev distance $\left(L_{\infty}\right)$ compromise programming minimization, so that the Pareto optimal solution may be obtained.
\end{abstract}

L. C. Domínguez-Dafauce $(\square)$

Escuela Politécnica, Universidad Europea de Madrid,

Madrid, Spain

e-mail: lcdd@nsp3.org

F. M. Martín

Termodinámica, Escuela Técnica Superior de Ingenieros de

Montes, Universidad Politécnica de Madrid, Madrid, Spain
Keywords Renewable and sustainable energy planning . Developing countries - Multi-criteria decision making · Avoided emissions · Global warming

$\begin{array}{ll}\text { Abbreviations } \\ \text { AC } & \text { Alternating current } \\ \text { BT } & \text { Biomass thermal } \\ \text { BEG } & \text { Biomass electricity generation } \\ \text { GDP } & \text { Gross domestic product } \\ \text { GOSL } & \text { Government of Sri Lanka } \\ \text { HH } & \text { Household } \\ \text { HMDP } & \text { High materially developed countries } \\ \text { IAEA } & \text { International Atomic Energy Agency } \\ \text { LEC } & \text { Levelized energy cost } \\ \text { L } & \text { k-dimensional distance } \\ \text { LMDC } & \text { Low materially developed countries } \\ \text { LPC } & \text { Linear programming computation } \\ \text { LRY } & \text { Real GDP per capita } \\ \text { MCDM } & \text { Multi-criteria decision making } \\ \text { MDG } & \text { Millennium development goals } \\ \text { MMTCDE } & \text { Million metric tons of carbon dioxide } \\ & \text { equivalents } \\ \text { MSW } & \text { Municipal solid waste } \\ \text { NCRE } & \text { Non-conventional renewable energy } \\ \text { PH } & \text { Pico hydro } \\ \text { PUCSL } & \text { Public Utilities Commission of Sri Lanka } \\ \text { PV } & \text { Photovoltaic system } \\ \text { RE } & \text { Renewable energy } \\ \text { SH } & \text { Small hydro } \\ \text { SHS } & \text { Solar home systems } \\ \text { SL } & \text { Sri Lanka } \\ \text { SLSEA } & \text { Sri Lanka Sustainable Energy Authority } \\ \text { SRIME } & \text { Sustainable and renewable implementation } \\ & \text { multi-criteria energy } \\ \text { UN } & \text { United Nations } \\ & \end{array}$


WP

WPP

Wind power

Wind power plant

\section{Introduction}

The MCDM has been used over the years [1, 2], and is indeed a currently widely used tool for energy applications [3-6]. The aim of this paper is to focus on a set of sustainable and renewable factors that will be assessed through a precedence constraints evaluation. The optimal solution will be then chosen among the possible solutions for every one of the six equations, applying the Linear Programming Computation (LPC). The best compromised solution is then found among the Pareto optimal solutions [7], which will allow rejection of the solutions corresponding to any of the six optimizing equations that are found to be situated furthest from the rest of the optimal values obtained for the remaining equations. This way the dominating solutions will be softened, letting energy planners base their decisions on a solution that can not be improved without making at least one of the variables worse off.

This method's greatest challenge is how investigators and then planners will decide to calculate the weights to be applied to every one of the six equations. This decision will require a broad consensus among a wide range of experts so that decisions are not postponed, generating conflict, wasting time and, therefore, damaging future programming.

The subjective part of the compromise programming is also a demanding issue, which has to be carefully and professionally performed. The value given to the different subjects must be thoroughly assessed, avoiding a personal opinionated view from the experts.

\section{SRIME model}

Figure 1 shows the steps that are proposed in this model, which has been specifically designed for low materially developed countries. The first stage is to carry out a thorough evaluation of the current energy demand, and subsequent future needs. Secondly, the possible renewable solutions will be assessed, taking into account the incountry possibilities, along with the international support, aiming to enunciate the first function, using the precedence constraints. ${ }^{1}$ The third step will be to study the potentially avoidable emissions so that the corresponding three

\footnotetext{
1 The authors are aware of the fact that there are many other optimization methods, like the cascade optimization or the ideal point discriminant analysis [1], and have indeed checked the obtained results using some of the mentioned methods, obtaining similar results.
}

functions may be stated $\left(F_{2}, F_{3}, F_{4}\right)$. Then the fifth function will come from a deep study of the possible interactions between health and education and renewable energy, according to the parameters described by the UN and other international organizations. The last phase is to enunciate the cost function $\left(F_{6}\right)$.

The $L_{\mathrm{k}}$ distances will then be minimized, according to the chosen weights, so that a compromised solution may be selected among the several obtained by the preferred optimization tool. $^{2}$

Functions

Maximization of $R E$ potential capacity: $F_{1}$

The so-called green economy focuses on the various advantages our world shall enjoy if we were to go green [811]. Not only climate change is involved here but also the potential enhancement of the overall development factors, especially the health co-benefits. You may find the corresponding equation and Table 1 below, which includes a summary of the potential precedence constraints the authors have chosen to be applied [12-14]. ${ }^{3}$

$$
\begin{aligned}
& F_{1}\left(x_{11}, x_{12}, \ldots, x_{i j}, \ldots, x_{\mathrm{nm}}\right) \\
& \quad=A_{11} x_{11}+A_{12} x_{12}+\cdots+A_{\mathrm{nm}} x_{\mathrm{nm}}
\end{aligned}
$$

\section{Environmental impact minimization: $F_{2}, F_{3}, F_{4}$}

Environmental impact has ben a major concern in the world for the past ten years. Local researchers are indeed looking into potential present and future sustainable possibilities $[15,16]$.

These are the three corresponding functions:

$$
\begin{aligned}
& F_{2}\left(x_{11}, x_{12}, \ldots, x_{i j}, \ldots, x_{\mathrm{nm}}\right) \\
& \quad=B_{11} x_{11}+B_{12} x_{12}+\cdots+B_{\mathrm{nm}} x_{\mathrm{nm}} \\
& \quad F_{3}\left(x_{11}, x_{12}, \ldots, x_{i j}, \ldots, x_{\mathrm{nm}}\right) \\
& \quad=C_{11} x_{11}+C_{12} x_{12}+\cdots+C_{\mathrm{nm}} x_{\mathrm{nm}} \\
& \quad F_{4}\left(x_{11}, x_{12}, \ldots, x_{i j}, \ldots, x_{\mathrm{nm}}\right) \\
& \quad=D_{11} x_{11}+D_{12} x_{12}+\cdots+D_{\mathrm{nm}} x_{\mathrm{nm}}
\end{aligned}
$$

$B_{i j} / C_{i j} / D_{i j}$ : life cycle $\mathrm{CO}_{2} / \mathrm{NO}_{\mathrm{x}} / \mathrm{SO}_{2}$ avoided emissions (ton/energy unit)

For all $B_{i j} \geq 0 ; C_{i j} \geq 0 ; D_{i j} \geq 0 \Rightarrow \max F_{2} ; F_{3} ; F_{4}-$ optimal avoided emissions maximization

\footnotetext{
${ }^{2}$ Please note subscript $\mathrm{i}$ denotes every RE type, and $j$ denotes the different sectors; i.e., $x_{i j}$ denotes the amount of fossil fuel (ktoe) replaced by RE $i$ in sector $j$.

${ }^{3}$ Coefficients A are non-dimensional and obtained through precedence constraints, where no energy or cost quantification is involved. These precedence constraints are based on the factors showed below in Table 1.
} 

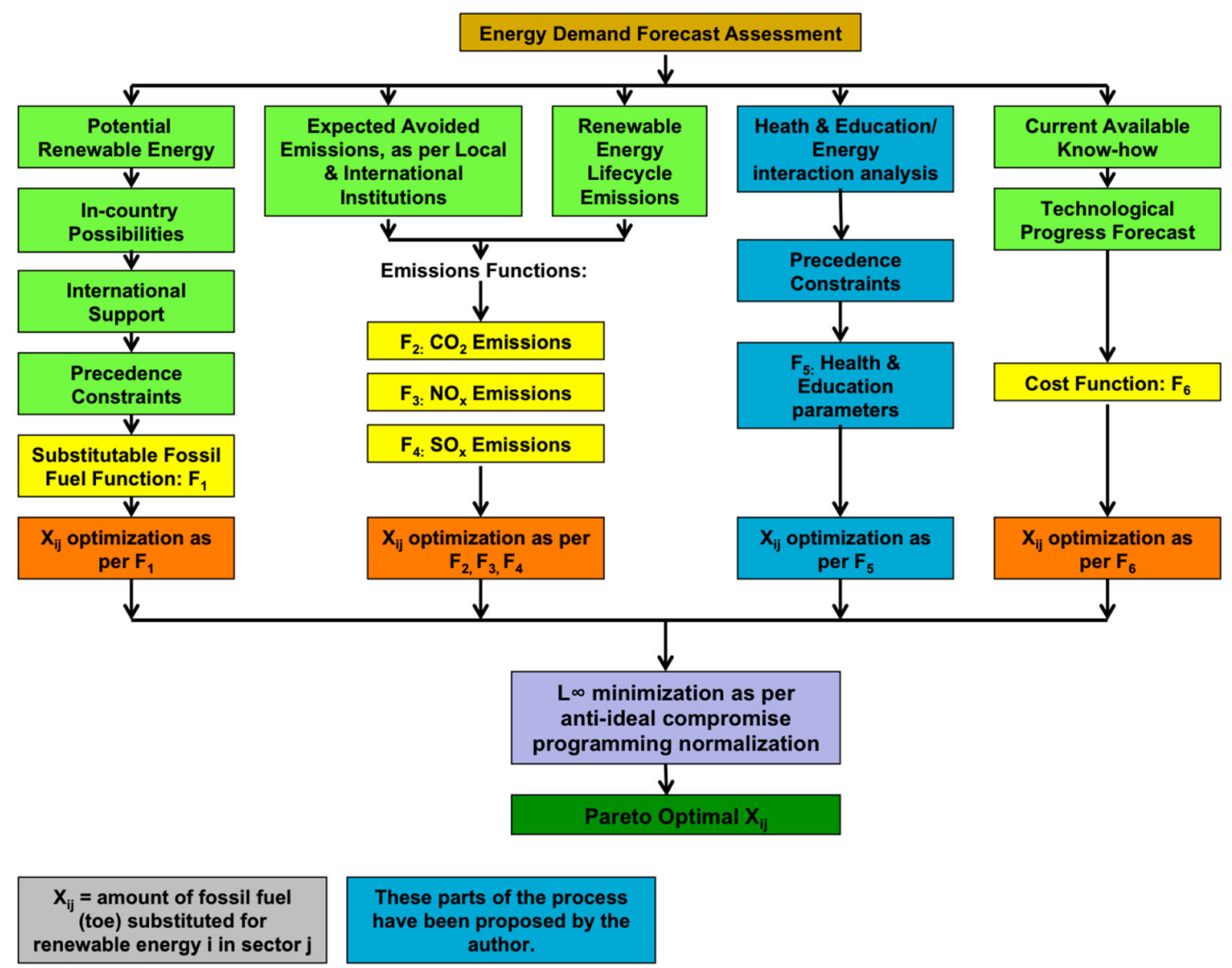

Fig. 1 SRIME energy model. Own construction

Table 1 Precedence constraints

\begin{tabular}{|c|c|c|c|}
\hline Technological factors & Application & Energy planning & $\begin{array}{l}\text { Fossil fuel } \\
\text { environmental } \\
\text { impact }\end{array}$ \\
\hline Technological demand & Operating time & Political interest & $\begin{array}{l}\text { Fossil fuel } \\
\text { environmental } \\
\text { impact }\end{array}$ \\
\hline Potential investigation & $\begin{array}{l}\text { Implementation } \\
\text { issues }\end{array}$ & $\begin{array}{l}\text { Current price compared } \\
\text { with conventional } \\
\text { energies }\end{array}$ & \\
\hline Know-how up-to-date & $\begin{array}{l}\text { Integration } \\
\text { possibility }\end{array}$ & Social demand & \\
\hline $\begin{array}{l}\text { Technology improvement (possibly } \\
\text { locally manufactured equipment) }\end{array}$ & Potential demand & Legislative forecast & \\
\hline $\begin{array}{l}\text { Current technological level } \\
\text { to apply the alternative energy }\end{array}$ & Supply availability & & \\
\hline
\end{tabular}

Own construction

high degree of causality. However, while HMDP show a

Coefficients $B_{i j}, C_{i j}, D_{i j}$ are obtained from official statistics and specialized publications.

\section{Maximization of the most rural development friendly types} of RE, aiming to improve health and education

bidirectional causality, LMDP only dictate a uni-directional causality from LRY to LEC [17]. As you can see below in Fig. 2, this is confirmed by the SL official data.

This is the corresponding equation:

One of the most usual indicators to estimate energy demand is the GDP growth rate. LEC and LRY commonly enjoy a

$$
\begin{aligned}
& F_{5}\left(x_{11}, x_{12}, \ldots, x_{i j}, \ldots, x_{\mathrm{nm}}\right) \\
& \quad=E_{11} x_{11}+E_{12} x_{12}+\ldots+E_{\mathrm{nm}} x_{\mathrm{nm}}
\end{aligned}
$$


Fig. 2 SL expenditure vs energy consumption. Prepared by the authors based on official data [18-23]

\section{Sri Lanka historical expenditure vs electricity cunsumption}

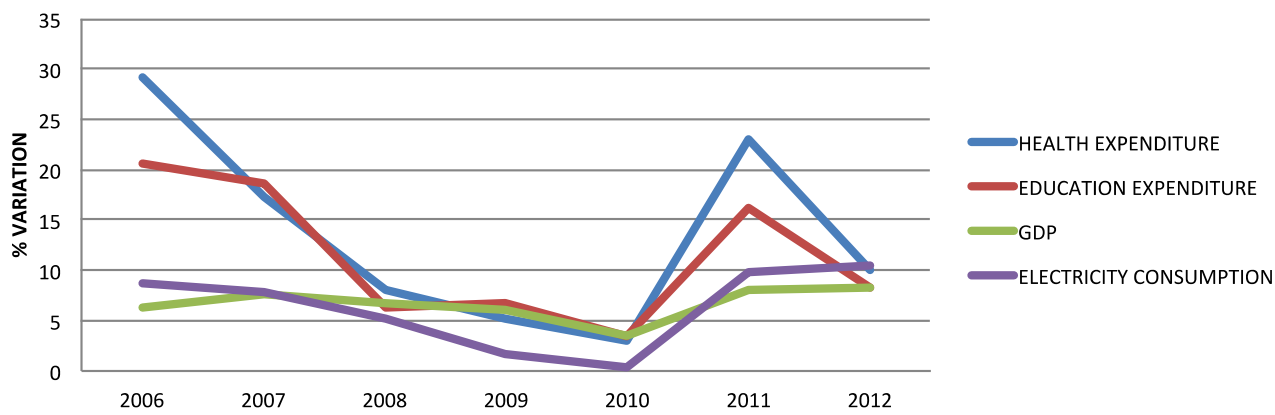

Coefficients $E_{i j}$ are non-dimensional and obtained through precedence constraints, where no energy or cost quantification is involved. These precedence constraints are based on:

1. Identified health and education ad-hoc applications.

2. Low overnight capital cost/unnecessary donor support for health and education enhancing applications.

3. Low maintenance cost.

4. Unnecessary maintenance contract.

5. Long lasting systems for health and education purposes.

6. Short distance from health and education centres to energy source.

7. Safe from being stolen in rural environment.

8. Low rural environmental impact.

9. Predictability.

10. Batteries not required for health and education purposes.

11. Alternating current.

12. Possible modularity/size accommodation for schools and hospitals.

13. Added benefits for rural development.

14. In-country development: manufacturing, training, etc.

15. Low LEC.

16. Hospitals and schools space efficiency for rural development.

17. Low or inexistent waste generation.

Cost minimization of substitution of renewable for existing conventional energy: $F_{6}$

$F_{6}\left(x_{11}, x_{12}, \ldots, x_{i j}, \ldots, x_{\mathrm{nm}}\right)=G_{11} x_{11}+G_{12} x_{12}$

$+\cdots+G_{\mathrm{nm}} x_{\mathrm{nm}}$

$G_{i j}=$ initial investment or production cost/energy unit

$G_{i j} \geq 0$ or $0 \leq G_{i j}$.

This function can also consider the renewable energy subsidies, therefore affecting the $C$ coefficients. Another possibility can be to modify the coefficients, aiming to reach the best possible subsidy for every RE.
As per coefficients $B_{i j}, C_{i j}$ and $D_{i j}$, coefficients $G_{i j}$ are also obtained from official sources.

\section{Restrictions}

The restrictions to be applied are described below in Table 2, having in mind the following [1, 3, 12 and own construction]:

$S_{j}$ : $\quad$ Energy demand of application $j$, calculated as: $S_{j}=p_{j}-i_{j}$; where $p_{j}$ is the energy demand corresponding to sector $j$ and $i_{j}$ is the amount of fossil fuel renewable sources can not replace.

$S_{\mathrm{e}}$ : Energy demand of application $i$, calculated as: $S_{\mathrm{e}}=p_{\mathrm{e}}-i_{\mathrm{e}}$; where $p_{\mathrm{e}}$ is the energy demand corresponding to sector $e$ and $i_{\mathrm{e}}$ is the amount of fossil fuel renewable sources can not replace.

$P_{i}$ : Potential application of renewable source $i$ in the corresponding sector.

$R_{i}$ : $\quad$ Minimum amount of conventional energy renewable sources can replace, as RE has already replaced this amount.

Compromise programming

As indicated by Linares et al. [24-26], to obtain the set of compromised solutions, both the normalized Manhattan distance, $L_{1}$ or the Chebyshev distance, $L_{\infty}$, may be

Table 2 Restrictions

\begin{tabular}{llll}
\hline $\begin{array}{l}\text { Non-electric } \\
\text { energy use }\end{array}$ & $\begin{array}{l}\text { Electric } \\
\text { energy use }\end{array}$ & $\begin{array}{l}\text { RE potential } \\
\text { production }\end{array}$ & $\begin{array}{l}\text { Current RE } \\
\text { systems }^{\mathrm{a}}\end{array}$ \\
\hline$\sum_{j=1}^{n} X_{i j} \leq S_{j}(7)$ & $\sum_{i=1}^{l} X_{i \mathrm{e}} \leq S_{\mathrm{e}}$ & $X_{i \mathrm{e}}+\sum_{j=1}^{m} X_{i j} \leq P_{i}$ & $X_{i \mathrm{e}}+\sum_{j=1}^{m} X_{i j} \leq R_{i}$ \\
& $(8)$ & $(9)$ & $(10)$
\end{tabular}

Own construction

a This restriction may also establish certain priorities in terms of advising the type of plant to be planned, always respecting the previous restrictions 
Table 3 Normalized distances to be minimized

\begin{tabular}{llc}
\hline$L_{1}$ & $L_{\mathrm{k}}$ & $L_{\infty}$ \\
\hline$L_{1}=\sum_{i=1}^{3} W_{i} \frac{F_{i}^{*}+-F_{i}(x)}{F_{i}^{*}+-F * i}$ & $\begin{array}{c}L_{k}=\lambda\left[\frac{W_{1} f_{1}(x)}{F_{1}^{*}+-F * 1}+\frac{W_{2} f_{2}(x)}{F_{2}^{*}+-F * 2}+\frac{W_{3} f_{3}(x)}{F_{3}^{*}+-F * 3}\right] \\
(12)\end{array}$ & $\begin{array}{c}\operatorname{Min} L_{\infty} \\
=\end{array}$ \\
\hline
\end{tabular}

Own construction

minimized [27, 28]. Nonetheless, $L_{\mathrm{k}}$ will also help us obtain the optimal solution. Equation (11) below shows the general definition for $L_{\mathrm{d}}$. Equations (12), (13) and (14) show the normalization to be carried out and Table 3 the distances that must be minimized.

$d=\left[\sum_{j=1}^{n}|x 1 j-x 2 j|^{d}\right]^{1 / d}$

Taking into account that $W_{\mathrm{i}}$ is the weight or preference assigned by the decision makers, the distances to be minimized may be found below in Table 4.

\section{Case study: Sri Lanka}

The authors have prepared a sectorial energy consumption forecast for 2015 (see Table 5; Fig. 3) and the estimated generation and capacity mix (see Figs. 4, 5). The global electricity data has been obtained from the Long Term Generation Expansion Plan 2013-2032, prepared by the Ceylon Electricity Board, and the rest of he energy consumption and the electricity sectorial data has been estimated by the authors based, on official and private documentation [29-50].

\section{The optimization equations}

$F_{1}$. Maximization of RE potential capacity

SL is very much dependent on petroleum, which currently accounts for approximately $24 \%$ of SL import bill and $45 \%$ of exports. The demand has been doubled, in value terms, during the last 3 years [52]. The geo-climatic settings are particularly conducive, though, to harnessing hydro resources. The climate is largely determined by the meteorological conditions caused in the Indian sub continent due to the tropical circulation [53]. The current hydro power stations are operated to meet both peak and base electricity generation requirements. A $400 \mathrm{MW}$ potential has been identified for small hydropower projects [54], typically characterised with less than $10 \mathrm{MW}$ capacities [53]. 128 projects, totalling $271 \mathrm{MW}$, have been already commissioned as of 31/12/2014 [55]. Once these projects are available for power generation, will be carefully followed-up by PUCSL [56]. In terms of wind power, GOSL would like to go from the current $5 \%$ to reach $10 \%$ by 2017 and $14.1 \%$ by 2022 [56]. There are close to $500 \mathrm{~km}^{2}$ of windy areas with good-to-excellent wind resource potential. However, only a portion of this is deemed feasible to be harnessed because of technical and system limitations [57]. As of 31/12/2013, there are 10 commissioned projects, which will add 78.45 MW capacity to the grid [55] and also some possible future WPPs [58-61]. The wind potential has been estimated as 20,000 MW. In terms of biomass, Gliricidia sepium has been recently appointed as the fourth plantation crop after tea, rubber and coconut. Biomass application in electricity generation is not yet

Table 4 Restrictions summary

\begin{tabular}{llll}
\hline Distance & $\begin{array}{l}d=1 \text { : Manhattan } \\
\text { distance, } L_{1}\end{array}$ & $\begin{array}{l}d=2 \text { : Euclidean } \\
\text { distance, } L_{2}\end{array}$ & $\begin{array}{l}d=\infty \text { : Chebyshev } \\
\text { distance, } L_{\infty}\end{array}$ \\
\hline Restrictions & $\sum_{j=1}^{n} X_{i j} \leq S_{j}(15)$ & $\sum_{j=1}^{n} X_{i j} \leq S_{j}(15)$ & $\sum_{j=1}^{n} X_{i j} \leq S_{j}(15)$ \\
$\sum_{i=1}^{l} X_{i \mathrm{e}} \leq S_{\mathrm{e}}(16)$ & $\sum_{i=1}^{l} X_{i \mathrm{e}} \leq S_{\mathrm{e}}(16)$ & $\sum_{i=1}^{l} X_{i \mathrm{e}} \leq S_{\mathrm{e}}(16)$ \\
$X_{i \mathrm{e}}+\sum_{j=1}^{m} X_{i j} \leq P_{i}(17)$ & $X_{i \mathrm{e}}+\sum_{j=1}^{m} X_{i j} \leq P_{i}(17)$ & $X_{i \mathrm{e}}+\sum_{j=1}^{m} X_{i j} \leq P_{i}(17)$ \\
& $X_{i \mathrm{e}}+\sum_{j=1}^{m} X_{i j} \leq R_{i}(18)$ & $X_{i \mathrm{e}}+\sum_{j=1}^{m} X_{i j} \leq R_{i}(18)$ & $X_{i \mathrm{e}}+\sum_{j=1}^{m} X_{i j} \leq R_{i}(18)$ \\
& $W_{1} \frac{F_{1}^{*}+-F_{1}(x)}{F_{1}^{*}+-F * 1} \leq D(19)$ & $W_{1} \frac{F_{1}^{*}+-F_{1}(x)}{F_{1}^{*}+-F * 1} \leq D(19)$ \\
& $W_{2} \frac{F_{2}^{*}+-F_{2}(x)}{F_{2}^{*}+-F * 2} \leq D(20)$ & $W_{2} \frac{F_{2}^{*}+-F_{2}(x)}{F_{2}^{*}+-F * 2} \leq D(20)$ \\
& $W_{3} \frac{F_{3}^{*}+-F_{3}(x)}{F_{3}^{*}+-F * 3} \leq D(21)$ & $W_{3} \frac{F_{3}^{*}+-F_{3}(x)}{F_{3}^{*}+-F * 3} \leq D(21)$ \\
\hline
\end{tabular}


Table 52015 SL sectorial energy consumption forecast (ktoe) [29-50]

\begin{tabular}{|c|c|c|c|c|c|c|c|c|c|c|c|c|}
\hline & Electricity & LPG & Gasoline & Naphta & Avgas & Kerosene & Avtur & Diesel & Foil & Coal & Bagasse & Fwood \\
\hline Agriculture & & & & & & & & 3.1 & 8.6 & & & \\
\hline Industries & 372.4 & 36 & & & & 25.1 & & 85.6 & 180.7 & 83 & 65.1 & 1961 \\
\hline Transport & & 1.8 & 981.8 & 2.7 & 0.2 & & 486.5 & 1924 & 2.7 & & & \\
\hline Education & 3.9 & & & & & & & & & & & \\
\hline Religious & 6.7 & & & & & & & & & & & \\
\hline $\mathrm{HH}$ & 428.6 & 105.4 & & & & 143.1 & & & & & & 3739.2 \\
\hline Hotels & 20.9 & 1.9 & & & & & & & 8.3 & & & \\
\hline Street light & 14.6 & & & & & & & & & & & \\
\hline Health & 15.7 & 1.2 & & & & & & & 2.4 & & & \\
\hline Commercial & 236.1 & 137.1 & & & & 15.3 & & 14.7 & 13.8 & & & 427.8 \\
\hline
\end{tabular}

Fig. 32015 Sri Lanka sectorial energy consumption forecast [29-50]

\section{SL sectorial consumption forecast}

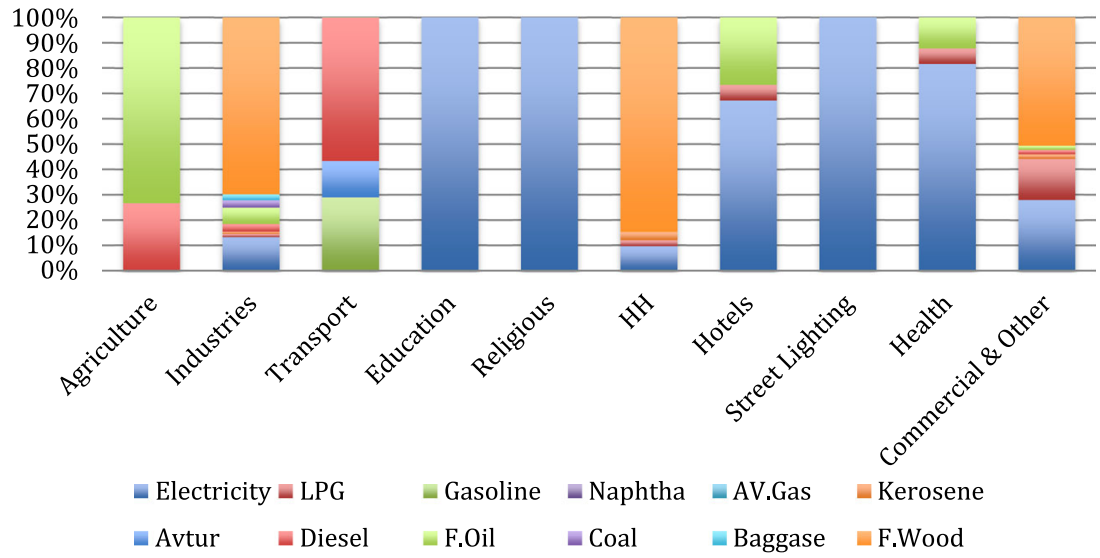

\section{SL 2015 estimated Generation Mix (GWh)}

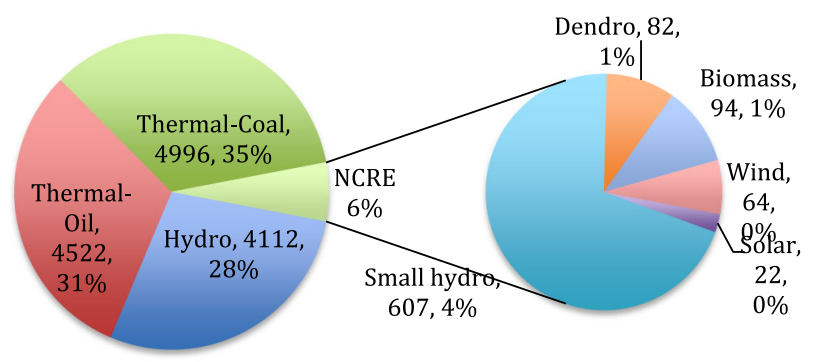

Fig. 4 SL 2015 estimated capacity mix [29-50]

widespread, but it is gaining momentum [54]. As of 31/12/ 2014, 2 Agricultural and Industrial Waste Power projects have been commissioned (11 MW); and another 2 Dendro Power (5.5 MW) [55]. A $1000 \mathrm{MW}$ of Dendro thermal potential has been estimated [59]. Biofuels are also planned to be developed to claim a 20 per cent share by 2020 [58,

\section{SL 2015 estimated Capacity Mix (MW)}

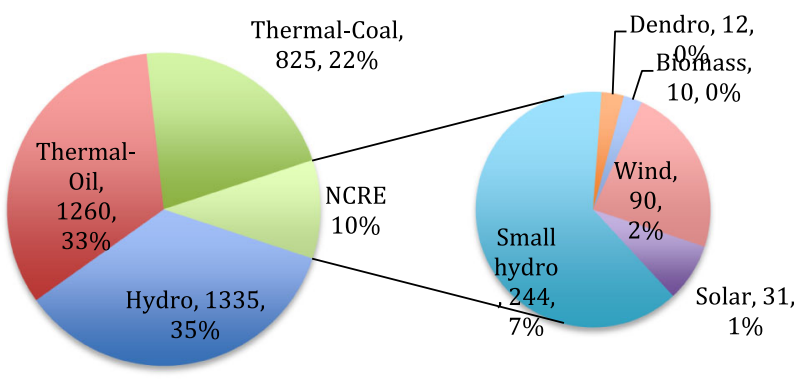

Fig. 5 SL 2015 estimated generation mix [29-51]

62]. Although the village biogas power is at an early stage, as it has not been an easy task to introduce it [63, 64], there are indeed a number of projects going on. This NCRE seems to be following the increasing tendency currently shown in South Asia [59]. A 300 MW MSW biogas generation potential has been identified [65]. 
As showed on Table 6 above, the use of SHSs has been spreading fast in the rural areas of Sri Lanka, mainly because of the financial incentives provided by the donor agencies, and also due to the aggressive marketing strategies of the SHS dealers in rural areas [66, 67]. As of 31/12/ 2013, 4 solar projects have been commissioned, totalling 1,4 MW. As of 31/12/2012, the installed PV capacity was $10.10 \mathrm{MWp}$ [68]. Concerning geothermal energy resources, SL is still at a preliminary stage, although a 700-1300 MWe potential has been recently estimated. As a first step in the development, a USD $10 \mathrm{M}$ investment would cover a site selection study, surface exploration at the most promising site followed by deep drilling, and commissioning of a $2 \mathrm{MW}$ binary power plant if the wells are successful [69]. Regarding off-grid schemes, a pilot project was recently conducted to connect two village micro hydro power plants (10 and $20 \mathrm{~kW}$ ) to the national grid. This pilot project has become instrumental in removing the technical, social, and legal barriers for grid interconnection. It is necessary, though, to review these fees structure and try to reduce them, taking into account the capacity of the project [70] and the potential funding [71-73]. The overall target for NCRE is to reach $10 \%$ by 2016 and $20 \%$ by 2020 [63]. Some authors are praising
SLs NCRE implementation [64], while others believe that lack of financing instruments, along with high initial cost and lack of assurance of resource supply or availability are the main barriers for renewable technologies expansion in SL $[65,66]$. Reaching the above mentioned targets is not just an environmental matter, but are related in one way or another to at least five other MDG [67, 74]:

1. Eradicate extreme poverty and hunger.

2. Achieve universal primary education.

3. Gender equality and empowering women.

4. Reduce child mortality.

5. Improve maternal health.

Taking into account all of the above, the authors have estimated that the following energy is considered to be substitutable in 2015 (see below Table 7).

The authors have defined the following variables:

- $\mathrm{BT}_{\mathrm{I}}$ : biomass thermal, industrial.

- $\mathrm{BT}_{\mathrm{H}}$ : biomass thermal, household.

- $\mathrm{BT}_{\mathrm{HE}}$ : biomass thermal, health.

- $\mathrm{BT}_{\mathrm{C}}$ : biomass thermal, commercial.

- $\mathrm{LBF}_{\mathrm{A}}$ : liquid biofuel, agriculture.

- $\mathrm{LBF}_{\mathrm{T}}$ : liquid biofuel, transport.

Table 62012 status of Sri Lanka off-grid energy technologies

\begin{tabular}{|c|c|c|c|c|c|}
\hline Technology & $\begin{array}{l}\text { Number of } \\
\text { units installed }\end{array}$ & $\begin{array}{l}\text { Capacity of a } \\
\text { unit }(\mathrm{KW})\end{array}$ & $\begin{array}{l}\text { Estimated total } \\
\text { capacity (MW) }\end{array}$ & Number of households electrified & $\begin{array}{l}\text { Number of units still } \\
\text { in use (estimated) }\end{array}$ \\
\hline Solar home systems (PV) & 120,000 & $0.03-0.06$ & 3.6 & 120,000 & 100,000 \\
\hline Village micro hydro power & 300 & $3-50$ & 4.5 & 10,500 & 80 \\
\hline Village dendro power & 11 & $10-30$ & 0.1 & 100 & - \\
\hline Wind home systems & 30 & $0.25-3.0$ & 0.007 & 30 & - \\
\hline Village biogas power & 10 & $0.3-2.0$ & 0.006 & 30 & 2 \\
\hline Pico hydro power & 40 & $0.3-1.5$ & 0.012 & 40 & 40 \\
\hline Total & & & 8.2 & 130,700 & \\
\hline
\end{tabular}

Data from Apergis and Payne [50]

Table 7 Substitutable energy in 2015 (ktoe)

\begin{tabular}{|c|c|c|c|c|c|c|c|c|c|c|c|c|}
\hline & Electricity & LPG & Gasoline & Naphta & Avgas & Kerosene & Avtur & Diesel & Foil & Coal & Bagasse & Fwood \\
\hline Agriculture & & & & & & & & 0.5 & 1.3 & & & \\
\hline Industries & 74.5 & & & & & & & 12.8 & 27.1 & & & \\
\hline Transport & & & 147.3 & & & & & 288.6 & 0.4 & & & \\
\hline Education & 0.8 & & & & & & & & & & & \\
\hline Religious & 1.3 & & & & & & & & & & & \\
\hline $\mathrm{HH}$ & 85.7 & & & & & & & & & & & \\
\hline Hotels & 4.2 & & & & & & & & 1.2 & & & \\
\hline Street light & 2.9 & & & & & & & & & & & \\
\hline Health & 3.1 & & & & & & & & 0.4 & & & \\
\hline Commercial & 47.2 & & & & & & & 2.2 & 2.1 & & & \\
\hline
\end{tabular}


- SH: small hydro.

- WPP: wind power plant.

- BEG: biomass for electricity generation.

- PV: photovoltaic electricity generation.

- MSW: municipal solid waste.

The restrictions for this case study have been established by the authors as per below:

A: Based on energy demand and RE real potential (in ktoe):

1. $\mathrm{LBF}_{\mathrm{A}} \leq 1.8$

2. $\mathrm{BT}_{\mathrm{I}} \leq 2148.9$

3. $\mathrm{LBF}_{\mathrm{T}} \leq 436.3$

4. $\mathrm{BT}_{\mathrm{H}} \leq 1.2$

5. $\mathrm{BT}_{\mathrm{HE}} \leq 0.4$

6. $\mathrm{BT}_{\mathrm{C}} \leq 432.1$

7. Electricity $^{4}$ :

$$
\begin{aligned}
\mathrm{SH} & +\mathrm{PV}+\mathrm{WPP}+\mathrm{MSW}+\mathrm{BEG} \leq 219.8 \\
\text { 8. } & \mathrm{SH} \leq 150 \\
\text { 9. } & \mathrm{PV} \leq 30 \\
\text { 10. } & \mathrm{WPP} \leq 100 \\
\text { 11. } & \mathrm{MSW} \leq 100 \\
\text { 12. } & \mathrm{BEG} \leq 60
\end{aligned}
$$

B: Based on the already existing RE (in ktoe):

1. $\mathrm{LBF}_{\mathrm{A}}, \mathrm{LBF}_{\mathrm{T}} \geq 0$

2. $\mathrm{BT}_{\mathrm{I}} \geq 2109^{5}$

3. $\mathrm{BT}_{\mathrm{H}} \geq 0$

4. $\mathrm{BT}_{\mathrm{HE}} \geq 0$

5. $\mathrm{BT}_{\mathrm{C}} \geq 427.8$

6. $\mathrm{SH} \geq 47.3$

7. $\mathrm{PV} \geq 3.6$

8. $\quad \mathrm{WPP} \geq 8.6$

9. $\mathrm{MSW} \geq 0$

10. $\mathrm{BEG} \geq 15.1$

To determine the coefficients corresponding to function $F_{1}$, the authors have established the precedence constraints included below in Table 8 .

The following values are then calculated (see Table 9 below):

$M_{i j}=\Pi_{i=1}^{n} a_{i j}$

$N_{i j}=\Pi_{i=1}^{n}\left(6-a_{i j}\right)$

Once the coefficients are applied, this is the final equation for $F_{1}$ :

\begin{tabular}{|c|c|c|c|c|c|c|c|}
\hline & BEG & BT & LBF & MSW & PV & $\mathrm{SH}$ & WPP \\
\hline $\begin{array}{l}\text { Technological } \\
\text { demand }\end{array}$ & 2 & 2 & 2 & 3 & 4 & 5 & 3 \\
\hline $\begin{array}{l}\text { Potential } \\
\text { investigation }\end{array}$ & 5 & 5 & 5 & 5 & 5 & 4 & 5 \\
\hline Know-how up-to-date & 4 & 5 & 3 & 4 & 4 & 3 & 5 \\
\hline $\begin{array}{l}\text { Technology } \\
\text { improvement } \\
\text { (possibly locally } \\
\text { manufactured } \\
\text { equipment) }\end{array}$ & 3 & 3 & 3 & 3 & 3 & 3 & 2 \\
\hline $\begin{array}{l}\text { Current technological } \\
\text { level to apply the } \\
\text { alternative energy }\end{array}$ & 2 & 3 & 2 & 2 & & & 4 \\
\hline Operating time & 3 & 5 & 2 & 3 & 3 & 4 & 2 \\
\hline Implementation issues & 3 & 5 & 2 & 3 & 3 & 5 & 3 \\
\hline Integration possibility & 5 & 5 & 5 & 5 & 5 & 5 & 5 \\
\hline Potential demand & 2 & 2 & 2 & 4 & 4 & 5 & 4 \\
\hline Supply availability & 3 & 3 & 2 & 4 & 4 & 4 & 4 \\
\hline Political interest & 2 & 2 & 2 & 3 & 3 & 5 & 4 \\
\hline $\begin{array}{l}\text { Current price } \\
\text { compared with } \\
\text { conventional } \\
\text { energies }\end{array}$ & 1 & 2 & 2 & 1 & 2 & 5 & 3 \\
\hline Social demand & 1 & 2 & 1 & 2 & 2 & 5 & 2 \\
\hline Legislative forecast & 3 & 3 & 3 & 3 & 3 & 3 & 3 \\
\hline Environmental impact & 5 & 3 & 5 & 5 & 5 & 5 & 5 \\
\hline
\end{tabular}

\footnotetext{
$\overline{4}$ Electricity is studied separately as per its special characteristics. To avoid being over-optimistic, a maximum $20 \%$ has been considered, having in mind that this percentage is expected by 2020 [47].

${ }^{5}$ Includes coal, bagasse and fuel wood as per Table 1.
}

Table 8 Precedence constraints [54, 75-103]

Table 9 Calculation of $N$ and $M$ factors

\begin{tabular}{llll}
\hline Alternative & $M$ & $N$ & Coefficient \\
\hline SH & 216 & $3.4 \times 10^{9}$ & 2.5 \\
WPP & 82,944 & $10^{8}$ & 2.5 \\
PV & 186,624 & $9 \times 10^{7}$ & 2 \\
BT & 248,832 & $2.4 \times 10^{7}$ & 2 \\
MSW & 466,560 & $2.3 \times 10^{7}$ & 2 \\
BEG & $3.1 \times 10^{6}$ & $1.9 \times 10^{6}$ & 1.5 \\
LBF & $8,847,360$ & 864,000 & 1 \\
\hline
\end{tabular}

Source: own construction

$$
F_{1}=2.5 \mathrm{SH}+2.5 \mathrm{WPP}+2 \mathrm{PV}+2 \mathrm{BT}_{\mathrm{I}}+2 \mathrm{BT}_{\mathrm{H}}
$$
$+2 \mathrm{BT}_{\mathrm{HE}}+2 \mathrm{BT}_{\mathrm{C}}+2 \mathrm{MSW}+1.5 \mathrm{BEG}+\mathrm{LBF}_{\mathrm{A}}+$ $\mathrm{LBF}_{\mathrm{T}}$

Environmental impact minimization

CEB expected generation system for 2015 [20] has been taken into account (see below Table 10) to establish the potentially avoided emissions. According to the different types of fuel and their corresponding GWh in 2015 , a 9.9 $\mathrm{tCO}_{2} /$ toe weighted average will be considered as the 
Table 10 Generation forecast data from the Long Term Generation Expansion Plan 2013-2032, prepared by the CEB [20]

\begin{tabular}{|c|c|c|c|c|c|c|}
\hline & \multicolumn{3}{|c|}{ Capacity balance (MW) } & \multicolumn{3}{|c|}{ Energy balance (GWh) } \\
\hline & 2013 & 2014 & 2015 & 2013 & 2014 & 2015 \\
\hline \multicolumn{7}{|l|}{ Plant name } \\
\hline \multicolumn{7}{|l|}{ Hydro } \\
\hline Existing major hydro & 1335 & 1335 & 1335 & 4112 & 4112 & 4112 \\
\hline New major hydro & 0 & 0 & 0 & 0 & 0 & 0 \\
\hline Mini hydro & 219 & 232 & 244 & - & - & - \\
\hline Sub total & 1554 & 1567 & 1579 & 4112 & 4112 & 4112 \\
\hline NCRE-wind & 90 & 90 & 90 & - & - & - \\
\hline NCRE-solar & 1.3 & 21 & 31 & - & - & - \\
\hline Total NCRE & & & & 613 & 658 & 692 \\
\hline \multicolumn{7}{|l|}{ Thermal existing and committed } \\
\hline Small gas turbines & 85 & 85 & 85 & 16 & 6 & 4 \\
\hline Diesel Sapugaskanda & 72 & 72 & 72 & 459 & 431 & 456 \\
\hline Diesel ext. Sapugaskanda & 72 & 72 & 72 & 491 & 485 & 487 \\
\hline Gas turbine no 7 & 115 & 115 & 115 & 290 & 250 & 286 \\
\hline Lakdhanavi & 0 & 0 & 0 & 0 & 0 & 0 \\
\hline Asia power & 49 & 49 & 49 & 334 & 309 & 332 \\
\hline KPS combined cycle & 165 & 165 & 165 & 550 & 454 & 549 \\
\hline AES combined cycle & 163 & 163 & 163 & 491 & 362 & 474 \\
\hline Colombo power & 60 & 60 & 0 & 419 & 417 & 0 \\
\hline ACE power Horona & 0 & 0 & 0 & 0 & 0 & 0 \\
\hline Ace power Matara & 0 & 0 & 0 & 0 & 0 & 0 \\
\hline Heladhanavi & 100 & 100 & 0 & 696 & 696 & 0 \\
\hline ACE power Embilipitiya & 100 & 100 & 0 & 692 & 672 & 0 \\
\hline Biomass (Dendro) & 10 & 10 & 10 & 94 & 94 & 94 \\
\hline Kerawalapitiya & 270 & 270 & 270 & 1325 & 935 & 1138 \\
\hline Puttalam coal & 275 & 550 & 825 & 1665 & 3241 & 4996 \\
\hline Northern power & 0 & 20 & 20 & 0 & 131 & 137 \\
\hline Chunnakkam power extension & 0 & 24 & 24 & 0 & 183 & 183 \\
\hline Sub total & 1535 & 1854 & 1870 & 7523 & 8668 & 9135 \\
\hline \multicolumn{7}{|l|}{ New thermal plants } \\
\hline Coal & 0 & 0 & 0 & 0 & 0 & 0 \\
\hline Gas turbine $75 \mathrm{MW}$ & 0 & 0 & 225 & 0 & 0 & 477 \\
\hline Gas turbine $105 \mathrm{MW}$ & 0 & 0 & 0 & 0 & 0 & 0 \\
\hline Coal trinco & 0 & 0 & 0 & 0 & 0 & 0 \\
\hline Biomass (Dendro) & 4 & 8 & 12 & 27 & 54 & 82 \\
\hline Sub total & 4 & 8 & 237 & 27 & 54 & 559 \\
\hline Total installed capacity & 3184 & 3540 & 3807 & - & - & - \\
\hline Installed capacity without $\mathrm{NCRE}^{\mathrm{a}}$ & 2874 & 3197 & 3442 & - & - & - \\
\hline Peak demand & 2451 & 2692 & 2894 & - & - & - \\
\hline Difference without $\mathrm{NCRE}^{\mathrm{a}}$ & 423 & 505 & 548 & - & - & - \\
\hline Difference $(\%)$ & 17.3 & 18.8 & 18.9 & - & - & - \\
\hline Total generation & - & - & - & 12,275 & 13,493 & 14,499 \\
\hline System demand & - & - & - & 12,296 & 13,508 & 14,513 \\
\hline Unserved energy & & & & 21 & 15 & 14 \\
\hline
\end{tabular}

${ }^{\text {a }}$ This installed capacity does not include the non-dispatchable NCRE, i.e., mini-hydro, wind and solar 
amount of avoided emission as well as $0.028 \mathrm{tNO}_{\mathrm{x}} /$ toe and $0.041 \mathrm{tSO}_{2}$ /toe [20, 104-114].

You can find below Table 11, which includes a summary of the life cycle emissions corresponding to the different types of renewable energy, based on the below mentioned references.

In terms of $\mathrm{LBF}, 90 \%$ of the $\mathrm{CO}_{2}$ emissions are considered to be potentially avoided [122-124] that is, approximately $2.7 \mathrm{tCO}_{2}$ /toe. When biofuel replaces gasoline and diesel in the transport sector, $\mathrm{SO}_{2}$ emissions are reduced, but changes in NOx emissions depend on the substitution pattern and technology. The effects of replacing gasoline with ethanol and biodiesel also depend on engine features. Biodiesel can have higher NOx emissions than petroleum diesel in traditional direct-injected diesel engines that are not equipped with NOx control catalysts [125]. This is why no NOx avoided emissions have been taken into account. A $50 \%$ average $\mathrm{SO}_{2}$ emissions reduction is however considered, as the avoided emissions will depend very much on the blend.

Please find below de corresponding functions:

$F_{2}=9.78 \mathrm{SH}+9.78 \mathrm{WPP}+9.44 \mathrm{PV}+9.27 \mathrm{BT}_{\mathrm{I}}+$ 9.27 $\mathrm{BT}_{\mathrm{H}}+9.27 \mathrm{BT}_{\mathrm{HE}}+9.27 \mathrm{BT}_{\mathrm{C}}+8.9 \mathrm{MSW}+9.38$ $\mathrm{BEG}+2.7 \mathrm{LBF}_{\mathrm{A}}+2.7 \mathrm{LBF}_{\mathrm{T}}$

$F_{3}=0.028 \mathrm{SH}+0.028 \mathrm{WPP}+0.026 \mathrm{PV}+0.02 \mathrm{BT}_{\mathrm{I}}$ $+0.02 \mathrm{BT}_{\mathrm{H}}+0.02 \mathrm{BT}_{\mathrm{HE}}+0.02 \mathrm{BT}_{\mathrm{C}}+0.028 \mathrm{MSW}+$ $0.02 \mathrm{BEG}$

$F_{4}=0.04126 \mathrm{SH}+0.0407 \mathrm{WPP}+0.038 \mathrm{PV}+0.041$

$\mathrm{BT}_{\mathrm{I}}+0.041 \mathrm{BT}_{\mathrm{H}}+0.041 \mathrm{BT}_{\mathrm{HE}}+0.041 \mathrm{BT}_{\mathrm{C}}+0.04128$

$\mathrm{MSW}+0.041 \mathrm{BEG}+0.0058 \mathrm{LBF}_{\mathrm{A}}+0.0058 \mathrm{LBF}_{\mathrm{T}}$

$\mathrm{F}_{5}$ : Cost minimization of substitution of RE for existing conventional energy

Based on the typical capital cost ranges for RE power generation technologies, USD/kW [49, 137-139], the overnight capital cost is calculated (USD/toe) so that the appropriate coefficients can be applied:

$$
F_{5}=0.08 \quad \mathrm{SH}+0.32 \quad \mathrm{WPP}+0.3 \quad \mathrm{PV}+0.18
$$

$\mathrm{BT}_{\mathrm{I}}+0.14 \quad \mathrm{BT}_{\mathrm{H}}+0.18 \quad \mathrm{BT}_{\mathrm{HE}}+0.14 \quad \mathrm{BT}_{\mathrm{C}}+0.48$ $\mathrm{MSW}+0.55 \mathrm{BEG}+0.94 \mathrm{LBF}_{\mathrm{A}}+0.94 \mathrm{LBF}_{\mathrm{T}}$

It must be noted that a CHP use has been assumed for MSW biogas plants and LBF production.

$\mathrm{F}_{6}$ : Maximization of the most rural development friendly types of RE, aiming to improve health and education

See below Table 12, where the precedence constraints have been evaluated by the authors as per the below mentioned subjects, based on the indicated references.

$\mathrm{M}$ and $\mathrm{N}$ are then calculated as per Eqs. (23) and (24) (see Table 13 below):
Table 11 RE life cycle $\mathrm{CO}_{2}$ emissions; $\mathrm{tCO}_{2} /$ toe, $\mathrm{tNO}_{\mathrm{x}} /$ toe, $\mathrm{tSO}_{2} /$ toe [109, 112-121]

\begin{tabular}{lllllll}
\hline $\begin{array}{l}\text { Life } \\
\text { cycle } \\
\text { emission }\end{array}$ & SH & WPP & PV & BT & MSW & BEG \\
\hline $\mathrm{CO}_{2}$ & 0.12 & 0.12 & 0.46 & 0.63 & 1 & 0.52 \\
$\mathrm{NO}_{\mathrm{X}}$ & 0.00006 & 0.0004 & 0.0017 & 0.0083 & 0.00009 & 0.0083 \\
$\mathrm{SO}_{2}$ & 0.00003 & 0.0005 & 0.003 & 0.00042 & 0.000015 & 0.00042 \\
\hline
\end{tabular}

Table 12 Precedence constraints [126-136]

\begin{tabular}{|c|c|c|c|c|c|c|}
\hline & $\begin{array}{l}\mathrm{P} \\
\text { and } \\
\mathrm{SH}\end{array}$ & SW & $\mathrm{B}$ & PV & LBF & MSW \\
\hline $\begin{array}{l}\text { Identified ad hoc } \\
\text { applications }\end{array}$ & 5 & 4 & 5 & 5 & 5 & 1 \\
\hline $\begin{array}{l}\text { Low overnight capital cost/ } \\
\text { unnecessary donor support }\end{array}$ & 2 & 3 & 5 & 1 & 1 & 1 \\
\hline Low maintenance cost & 4 & 5 & 4 & 5 & 1 & 1 \\
\hline $\begin{array}{l}\text { Unnecessary maintenance } \\
\text { contract }\end{array}$ & 4 & 1 & 5 & 4 & 1 & 1 \\
\hline Long lasting systems & 5 & 4 & 4 & 4 & 4 & 5 \\
\hline $\begin{array}{l}\text { Short distance from energy } \\
\text { source }\end{array}$ & 4 & 5 & 5 & 5 & 2 & 1 \\
\hline $\begin{array}{l}\text { Safe from being stolen in } \\
\text { rural environment }\end{array}$ & 4 & 4 & 3 & 1 & 5 & 5 \\
\hline $\begin{array}{l}\text { Low rural environmental } \\
\text { impact }\end{array}$ & 3 & 5 & 2 & 5 & 2 & 2 \\
\hline Predictability & 4 & 2 & 4 & 2 & 4 & 5 \\
\hline Batteries not required & 2.5 & 1 & 2.5 & 1 & 2.5 & 2.5 \\
\hline $\mathrm{AC}$ & 2.5 & 2.5 & 2.5 & 1 & 2.5 & 2.5 \\
\hline Possible rural modularity & 1 & 2.5 & 1 & 2.5 & 1 & 1 \\
\hline $\begin{array}{l}\text { Added benefits for rural } \\
\text { development }\end{array}$ & 1 & 1 & 1 & 1 & 1 & 2.5 \\
\hline In-country development & 5 & 3 & 3 & 1 & 5 & 5 \\
\hline Low LEC & 4 & 4 & 3 & 1 & 1 & 1 \\
\hline $\begin{array}{l}\text { Space efficiency for rural } \\
\text { Environment }\end{array}$ & 1 & 4 & 1 & 3 & 1 & 1 \\
\hline Low waste generation & $2.5 \mathrm{~s}$ & 2.5 & 1 & 2.5 & 2.5 & 1 \\
\hline
\end{tabular}

Table 13 Calculation of $N$ and $M$ factors

\begin{tabular}{llll}
\hline Alternative & $M$ & $N$ & Coefficient \\
\hline P and SH & $4.1 \times 10^{6}$ & $4.8 \times 10^{7}$ & 2.5 \\
SW & $6.2 \times 10^{6}$ & $3.6 \times 10^{7}$ & 2.5 \\
B & $6.6 \times 10^{6}$ & $1.35 \times 10^{7}$ & 2 \\
PV & $4.6 \times 10^{7}$ & $4 \times 10^{5}$ & 2 \\
LBF & $2 \times 10^{8}$ & $10^{5}$ & 1.5 \\
MSW & $3.3 \times 10^{8}$ & 19,531 & 1 \\
\hline
\end{tabular}

Own construction 
Table 14 Optimization results using Matlab $^{\circledR}$ programming tools
Own construction

a Weights have been indicated as per functions vector $\left(F_{1}, F_{2}\right.$, $F_{3}, F_{4}, F_{5}, F_{6}$ )

\begin{tabular}{lccccccccccc}
\hline Weights & $\mathrm{SH}$ & $\mathrm{WP}$ & $\mathrm{PV}$ & $\mathrm{BT}_{\mathrm{I}}$ & $\mathrm{BT}_{\mathrm{H}}$ & $\mathrm{BT}_{\mathrm{HE}}$ & $\mathrm{BT}_{\mathrm{C}}$ & $\mathrm{MSW}$ & $\mathrm{BEG}$ & $\mathrm{LBF}_{\mathrm{A}}$ & $\mathrm{LBF}_{\mathrm{T}}$ \\
\hline $1,1,1,1,1,1^{\mathrm{a}}$ & 86.7 & 43.2 & 3.6 & 2146.4 & 1.1 & 0.4 & 431.8 & 62.3 & 15.1 & 1 & 180.1 \\
$1,0,0,0,0,0$ & 125.9 & 75.2 & 3.6 & 2148.9 & 1.2 & 0.4 & 432.1 & 0 & 15.1 & 1.8 & 436.3 \\
$5,1,1,1,1,1$ & 109.1 & 61.7 & 3.6 & 2148.9 & 1.2 & 0.4 & 432.1 & 30.2 & 15.1 & 1.5 & 325.1 \\
$0,1,0,0,0,0$ & 125.2 & 75.9 & 3.6 & 2148.9 & 1.2 & 0.4 & 432.1 & 0 & 15.1 & 1.8 & 436.3 \\
$1,5,1,1,1,1$ & 104.4 & 58.1 & 3.6 & 2148.7 & 1.2 & 0.4 & 432.1 & 37.8 & 15.1 & 1.4 & 300.8 \\
$0,0,1,0,0,0$ & 78.3 & 36.3 & 3.6 & 2148.9 & 1.2 & 0.4 & 432.1 & 86.5 & 15.1 & 0.9 & 114.7 \\
$1,1,5,1,1,1$ & 86.7 & 43.5 & 3.6 & 2148.8 & 1.2 & 0.4 & 432.1 & 70.7 & 15.1 & 1.1 & 173.7 \\
$0,0,0,1,0,0$ & 47.3 & 86 & 3.6 & 2148.9 & 1.2 & 0.4 & 432.1 & 14.5 & 15.1 & 1.8 & 436.3 \\
$1,1,1,5,1,1$ & 95.5 & 50.7 & 3.6 & 2148.8 & 1.2 & 0.4 & 432.1 & 54.4 & 15.1 & 1.3 & 247.1 \\
$0,0,0,0,1,0$ & 47.3 & 8.6 & 3.6 & 2109 & 0 & 0 & 427.8 & 0 & 15.1 & 0 & 0 \\
$1,1,1,1,5,1$ & 61.2 & 20.8 & 3.6 & 2121.1 & 0.4 & 0.1 & 429.1 & 18 & 15.1 & 0.4 & 66.7 \\
$0,0,0,0,0,1$ & 125 & 76.1 & 3.6 & 2148.9 & 1.2 & 0.4 & 432.1 & 0 & 15.1 & 1.8 & 436.3 \\
$1,1,1,1,1,5$ & 112.6 & 64.6 & 3.6 & 2148.8 & 1.2 & 0.4 & 432.1 & 23.5 & 15.1 & 1.6 & 348.3 \\
$1,5,5,5,1,1$ & 104.3 & 58.2 & 3.6 & 2148.9 & 1.2 & 0.4 & 432.1 & 38.4 & 15.1 & 1.4 & 299.1 \\
$5,1,1,1,1,5$ & 112.6 & 64.4 & 3.6 & 2148.8 & 1.2 & 0.4 & 432.1 & 23.6 & 15.1 & 1.6 & 349.7 \\
\hline
\end{tabular}

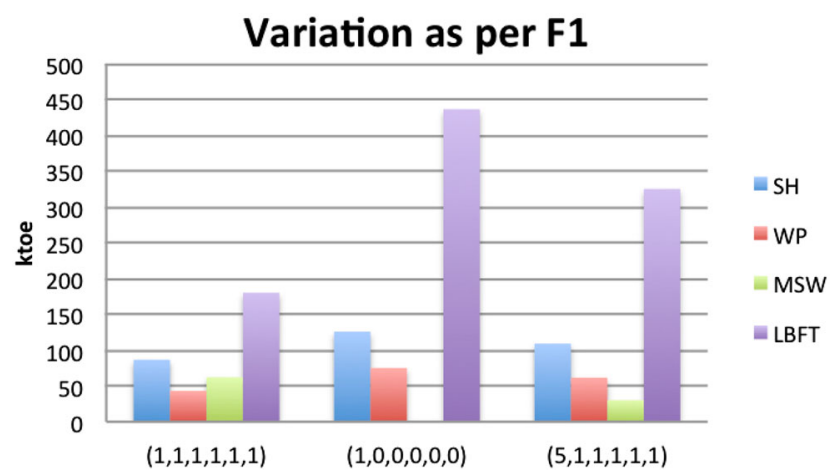

Fig. $6 \mathrm{SH}, \mathrm{WP}, \mathrm{MSW}$ and $\mathrm{LBF}_{\mathrm{T}}$ variation as per the weights assigned to function 1. Source: own construction

Once the coefficients are applied, this is the final equation for $\mathrm{F}_{6}$ :

$F_{6}=2.5 \mathrm{SH}+2.5 \mathrm{WPP}+2 \mathrm{PV}+2 \mathrm{BT}_{\mathrm{I}}+2 \mathrm{BT}_{\mathrm{H}}+2$ $\mathrm{BT}_{\mathrm{HE}}+2 \mathrm{BT}_{\mathrm{C}}+\mathrm{MSW}+2 \mathrm{BEG}+1.5 \mathrm{LBF}_{\mathrm{A}}+1.5 \mathrm{LBF}_{\mathrm{T}}$

\section{Results and discussion}

Please find below Table 14 and Figs. 6, 7, 8, 9, 10 and 11 , which include the results obtained (in ktoe), using the Chebyshev distance, L $\infty$, following the Anti-Ideal Compromise Programming as previously defined in

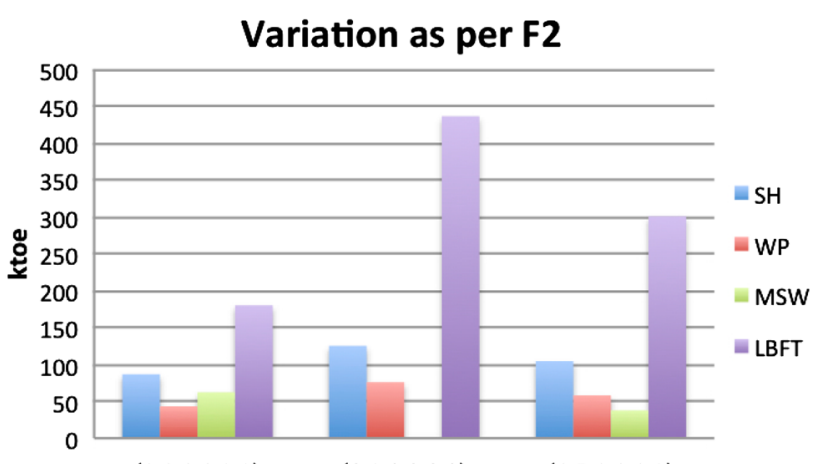

Fig. $7 \mathrm{SH}, \stackrel{(1,1,1,1,1,1)}{\mathrm{W} P}, \mathrm{MSW}$ and ${ }^{\mathrm{L}} \mathrm{L}^{\mathrm{B}} \mathrm{F}_{\mathrm{T}}$ variation ${ }^{(1,5,1,1,1,1)}$ as per the weights assigned to function 2. Source: own construction

chapter 2.3; taking into account the below mentioned weights.

The authors have aimed to compare the solution obtained given no special weight to any of the functions: $(1,1,1,1,1,1)$; with the obtained values when only one of the functions is taken into account: for example $(1,0,0,0,0,0)$; and finally with the outcome solution when a special weight has been given to a particular function: for example $(5,1,1,1,1,1)$. This way, the different solutions will clearly show the tendency the variables follow, when a particular function is given more importance than the others. The authors have added two more cases to this list, one giving special importance to the three emissions functions $\left(F_{2}, F_{3}\right.$, 


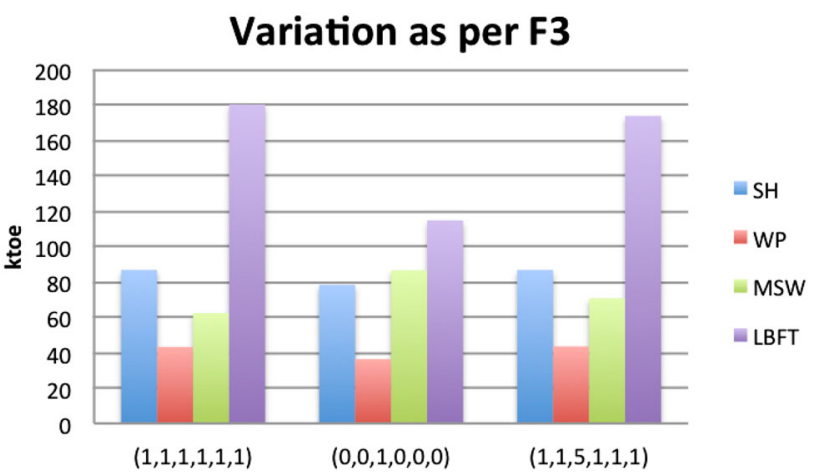

Fig. $8 \mathrm{SH}$, WP, MSW and $\mathrm{LBF}_{\mathrm{T}}$ variation as per the weights assigned to function 3 . Source: own construction

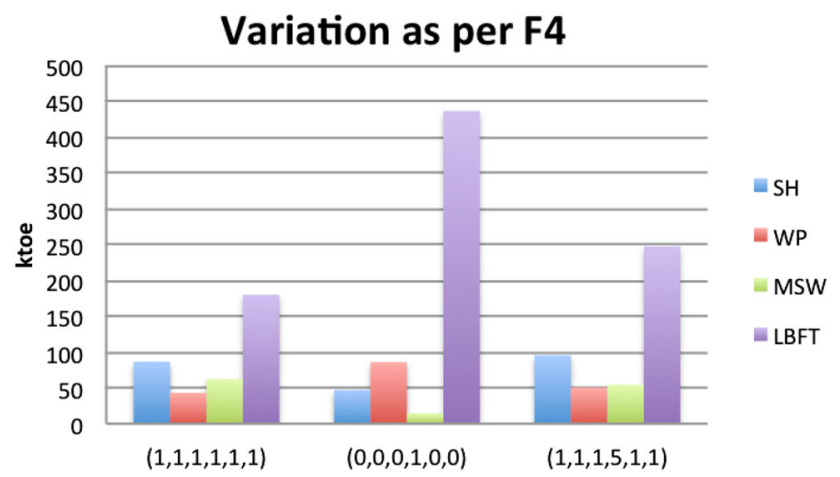

Fig. $9 \mathrm{SH}$, WP, MSW and $\mathrm{LBF}_{\mathrm{T}}$ variation as per the weights assigned to function 4. Source: own construction

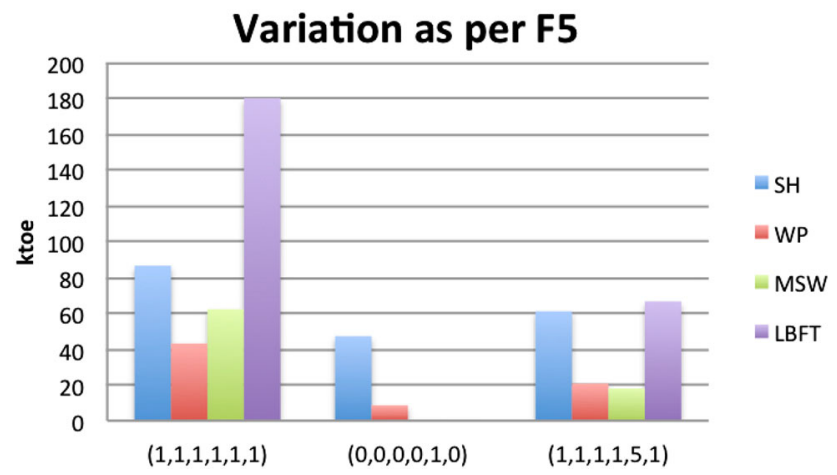

Fig. $10 \mathrm{SH}, \mathrm{WP}, \mathrm{MSW}$ and $\mathrm{LBF}_{\mathrm{T}}$ variation as per the weights assigned to function 5. Source: own construction

$F_{4}$ ), and another one targeting the maximum renewable energy substitution and rural health and education development $\left(F_{1}\right.$ and $\left.F_{6}\right)$.

The nominal weights $(1,1,1,1,1,1)$ are considered as the baseline. Once this baseline is obtained, then the other cases will be taken into account to give some special relative importance to any of the six functions or even to address some different weights to be applied if necessary $[15,16,23,24]$. The results show that PV and BEG stay at

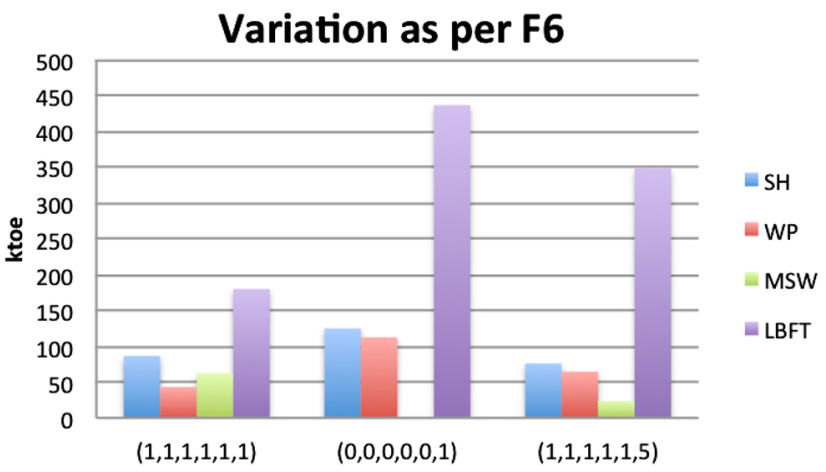

Fig. $11 \mathrm{SH}, \mathrm{WP}, \mathrm{MSW}$ and $\mathrm{LBF}_{\mathrm{T}}$ Variation as per the weights assigned to function 6 . Source: own construction

their minimum potential value independently of the chosen weights, while the four biomass variables $\left(\mathrm{BT}_{\mathrm{I}}, \mathrm{BT}_{\mathrm{H}}, \mathrm{BT}_{\mathrm{HE}}\right.$ and $\mathrm{BT}_{\mathrm{C}}$ ) reach approximately their maximum potential value, except for the two cases where the economic factor is given a certain weight versus the other functions. This exception is totally expected, as function $F_{5}$ will always look for the cheapest solution, i.e., the one implementing less renewable energy substitution. The rest of the variables, SH, WP, MSW LBF and LBFT do vary, depending on the chosen weights. The first three variables are linked together as per Eq. (22). This means that any variation in one of them, will therefore affect the other. As can be seen in Figs. 6, 7, 8, 9, 10 and 11 above, when only one function is optimized, a maximum polarized value is obtained for the different variables. Once all six functions are taking into account, even if a weight 5 is applied to a particular one, the difference from the nominal results [the ones obtained as per $(1,1,1,1,1,1)]$ is less acute, flattening this way the value given to the different variables.

If the individual optimizations [for example: $(1,0,0,0,0,0)]$ are not considered, and the last two cases or also not taken into account, the maximization of the above mentioned variables may be summarized as per Table 15 below.

The authors have summarized below in Table 16 the advantages of applying this energy planning model to Sri Lanka, and also the potential improvements in Table 17.

\section{Conclusions}

This paper shows the possibility of using MCDM methods, focusing on a sustainable and renewable energy approach. The resulting energy models give a great level of importance to the human environmental development factors, avoiding, therefore, the purely economic reasoning. These type of models are becoming a common way of choosing global energy plans [139] or some independent power 
Table 15 Maximization of SH, WP, MSW, $\mathrm{LBF}_{\mathrm{A}}$ and $\mathrm{LBF}_{\mathrm{T}}$

\begin{tabular}{ll}
\hline $\begin{array}{l}\text { Renewable } \\
\text { energy }\end{array}$ & Reaches maximum value when prioritizing \\
\hline $\mathrm{SH}$ & $\begin{array}{c}F_{6} \text { (max rural } \\
\text { development })>F_{1}>F_{2}>F_{4}>F_{3}>F_{5}\end{array}$ \\
$\mathrm{WP}$ & $\begin{array}{l}F_{6} \text { (max rural } \\
\text { development })>F_{1}>F_{2}>F_{4}>F_{3}>F_{5}\end{array}$ \\
$\mathrm{MSW}$ & $\begin{array}{c}F_{3} \text { (max NOx avoided } \\
\text { emissions) }>F_{4}>F_{2}>F_{1}>F_{6}>F_{5}\end{array}$ \\
$\mathrm{LBF}_{\mathrm{A}}$ & $\begin{array}{c}F_{6} \text { (max rural } \\
\text { development })>F_{1}>F_{2}>F_{4}>F_{3}>F_{5}\end{array}$ \\
$\mathrm{LBF}_{\mathrm{T}}$ & $\begin{array}{c}F_{6} \text { (max rural } \\
\text { development })>F_{1}>F_{2}>F_{4}>F_{3}>F_{5}\end{array}$ \\
&
\end{tabular}

Own construction

Table 16 Advantages of SRIME application in Sri Lanka

\begin{tabular}{|c|c|}
\hline SRIME singularity & Advantage for Sri Lanka \\
\hline $\begin{array}{l}\text { Multi-objective ( } 6 \\
\text { equations) }\end{array}$ & $\begin{array}{l}\text { Avoiding this way, single-objective } \\
\text { financial perspective, which gradually } \\
\text { would make SL poorer and more } \\
\text { dependent on oil import }\end{array}$ \\
\hline $\begin{array}{l}\text { Takes into account } \mathrm{CO}_{2} \\
\text { and also } \mathrm{NO}_{\mathrm{x}} \text { and } \mathrm{SO}_{2}\end{array}$ & $\begin{array}{l}\text { Automobiles are very contaminant in } \\
\mathrm{SL}, \mathrm{NO}_{\mathrm{x}} \text { is therefore of great } \\
\text { importance }\end{array}$ \\
\hline Maximizing RE & $\begin{array}{l}\text { SL has proofed to be a fast RE growing } \\
\text { country, improving industry in } \\
\text { general }\end{array}$ \\
\hline $\begin{array}{l}\text { Health and Education } \\
\text { equation }\end{array}$ & $\begin{array}{l}\text { Very innovative precedence constraints } \\
\text { application, aiming to set a } \\
\text { sustainable growth through energy } \\
\text { universal access }\end{array}$ \\
\hline $\begin{array}{l}\text { Possibility of applying } \\
\text { weights }\end{array}$ & $\begin{array}{l}\text { This way planners may choose which } \\
\text { either if all objectives may have the } \\
\text { same weight or there is a particular } \\
\text { equation that must be given a certain } \\
\text { priority }\end{array}$ \\
\hline Pareto optimal solution & $\begin{array}{l}\text { Assuring the chosen solution is not } \\
\text { 'dominated' by any of the equations }\end{array}$ \\
\hline
\end{tabular}

Own construction

plants, such as wind farms [140]. This work has aimed at establishing a potential energy model implementation methodology that may be applicable for developing countries. The Case Study shows that if SRIME was implemented in Sri Lanka, SH, WP, MSW and $\mathrm{LBF}_{\mathrm{T}}$ would be specially benefited from the Pareto optimization, while PV and BEG would stay at their official future expected value [20], having no growth at all. The SRIME model could be relatively easily interpolated to other Asian tropical countries, due to the similar circumstances in terms of health an
Table 17 Potential Improvements for SRIME application in Sri Lanka

\begin{tabular}{ll}
\hline Potential Improvement & Advantage \\
\hline Introduction of hybrid systems & $\begin{array}{c}\text { Very efficient for remote rural } \\
\text { materially underdeveloped } \\
\text { areas, with highly flexible } \\
\text { configurations, and currently } \\
\text { undergoing a high level of } \\
\text { implementation }\end{array}$ \\
$\begin{array}{ll}\text { Equations weights to be decided } \\
\text { by an international team of } \\
\text { specialists (energy planners, } \\
\text { maintenance specialists, policy } \\
\text { makers and politicians) }\end{array}$ & $\begin{array}{l}\text { processes of the SRIME is the } \\
\text { weights assignation. Although } \\
\text { these groups may have different } \\
\text { including, of course, people }\end{array}$ \\
points of view, and sometimes \\
even opposite objectives: \\
cri Lanka
\end{tabular}

Own construction

education low overall parameters, high biomass consumption, monsoon special weather characteristics, low GDP and extremely high petroleum imports dependency.

Acknowledgments The authors would like to gratefully acknowledge the support received from Carlos Molina-Dafauce. The authors would also like to thank professors Carlos Romero, Pedro Linares, Cristina Pascual, José L. Hernanz, Inés Izquierdo, and investigator Eder Falcón.

Conflicts of interest The authors declare no conflict of interest

Open Access This article is distributed under the terms of the Creative Commons Attribution License which permits any use, distribution, and reproduction in any medium, provided the original author(s) and the source are credited.

\section{References}

1. Marcos, F.: Aplicación de las técnicas multidimensionales a la planificación energética, pp. 97-104. Julio Agosto, Energía (1985)

2. Linares P.: Una aplicación de la programación multiobjetivo a la planificación eléctrica. CIEMAT-IEE. http://www.iit.upco millas.es/ pedrol/documents/energia97.pdf (2000). Accessed 5 October 2013

3. García L.: Desarrollo de un modelo multicriterio-multiobjetivo de oferta de energías renovables: aplicación a la comunidad de Madrid. Unpublished doctoral thesis. ETSI de Montes. UPM. Madrid (2004)

4. Williamsona, S.J., Starka, B.H., Bookerb, J.D.: Low head pico hydro turbine selection using a multi-criteria analysis. Renew. Energy 61, 43-50 (2014)

5. Gong, J., Xie, D., Jiang, C., Zhang, Y.: Multiple objective compromised method for power management in virtual power plants. Energies 4(4), 700-716 (2011) 
6. Maheri, A.: Multi-objective design optimisation of standalone hybrid wind-PV-diesel systems under uncertainties. Renew. Energy 66, 650-661 (2014)

7. Tomoiaga, B., Chindris, M., Sumper, A., Sudira-Andreu, A., Villafafila-Robles, R.: Pareto optimal reconfiguration of power distribution systems using a genetic algorithm based on NSGAII. Energies 6(3), 1439-1455 (2013)

8. REN21: Renewables 2005 Global Status Report. Washington DC. http://www.worldwatch.org/brain/media/pdf/pubs/ren21/ ren21-2.pdf (2005). Accessed 10 December 2013

9. REN21: Energy for development, the potential role of renewable energy in meeting the millennium development goals. http:// www.worldwatch.org/system/files/ren21-1.pdf (2005). Accessed 13 December 2013

10. Senadeera, R., Dissanayake, L.: Sri Lanka: towards a renewable energy island. In: Sustainable Future Energy 2012 and 10th SEE Forum Innovations for Sustainable and Secure Energy. Brunei Darussalam (2012)

11. World Health Organization (WHO): Health in the green economy, co-benefits to health of climate change mitigationhousing sector. http://www.who.int/hia/hgehousing.pdf (2011). Accessed 10 December 2013

12. Kydes, A.S.: The Brookhaven Energy System Optimization Model: its variants and uses. Energy Policy Modelling: United States and Canadian Experiences, pp 110-136 (1980)

13. Integrating Renewable Electricity on the Grid: American Physical Society (APS), Panel on Public Affairs (POPA). http:// www.aps.org/policy/reports/popa-reports/upload/integratingelec. pdf (2010). Accessed 20 Nov 2013

14. Sri Lanka Energy Sustainable Energy Authority: renewable energy policy targets. http://www.energy.gov.lk/sub_pgs/ energy_renewable_intro_policy.html. Accessed 25 Nov 2013

15. Wijayatunga, P.D., Fernando, W.J.L.S., Shrestha, R.M.: Greenhouse gas emission mitigation in the Sri Lanka power sector supply side and demand side options. Energy Convers. Manag. 44, 3247-3265 (2003)

16. Wijayatunga, P.D., Prasad, D.: Clean energy technology and regulatory interventions for Greenhouse Gas emission mitigation: Sri Lankan power sector. Energy Convers. Manag. 50, 1595-1603 (2009)

17. Lee, C.C., Chang, C.P.: Energy consumption and GDP revisited: a panel analysis of developed and developing countries. Energy Econ. 29, 1206-1223 (2007)

18. World Health Organization (WHO): Countries: Sri Lanka. Statistics. http://www.who.int/countries/lka/en/ (2013). Accessed 20 Nov 2013

19. Central Bank of Sri Lanka: Economic and Social Statistics of Sri lanka 2013. Central Bank of Sri Lanka, vol. XXXIV. http:// www.cbsl.gov.lk/pics_n_docs/10_pub/_docs/statistics/other/econ _\&_ss_2013_e.pdf (2013). Accessed 10 Jan 2014

20. Ceylon Electricity Board, Transmission and Generation Planning Branch Transmission Division: Long Term Generation Expansion Plan 2013-2032. http://www.pucsl.gov.lk/english/ wp-content/uploads/2013/11/LTGEP\%202013-2032.pdf (2012)

21. Ceylon Electricity Board: Colombo. http://www.ceb.lk/sub/pub lications/statistical.aspx. Accesed 11 Jan 2014

22. United Nations: World Economic Situation and Prospects 2014, Update as of mid-2014 (2014)

23. Mumtaz, R., Zaman, K., Sajjad, F., Lodhi, M.S., Irfan, M., Khan, I., Naseem, I.: Modeling the causal relationship between energy and growth factors: journey towards sustainable development. Renew. Energy 63(2014), 353-365 (2014)

24. Linares, P.: Integración de Criterios Medioambientales en Procesos de Decisión: Una Aproximación Multicriterio a la Planificación Integrada de Recursos. Unpublished doctoral thesis. E.T.S.I. de Montes. U.P.M. Madrid (1999)
25. Zeleny, M.: Compromise programming, in: Múltiple Criteria Decisión Making. Univertity of South Carolina Press, Columbia (1973)

26. Yu, P.L.: A class of solutions for group decision problems. Manag. Sci. 19(1973)

27. Romero, C.: Análisis de las decisiones multicriterio. Isdefe Madrid. ftp://ece.buap.mx/pub/DOCUM_EDUCATIVOS_FCE_ F_PORRAS/PROCESOS $\% 20$ DE\%20PENSAMIENTO $\% 20 \mathrm{y} \% 20$ TOC/teor\%EDa\%20generl\%20de\%20sistemas/Decisiones.pdf (1996). Accessed 10 Oct 2013

28. Romero, C.: Teoría de la decisión multicriterio: Conceptos, técnicas $\mathrm{y}$, aplicaciones edn. Alianza Universidad Textos, Madrid (1993)

29. Central Bank of Sri Lanka: Economic and Social Statistics of Sri Lanka 2013. Central Bank of Sri Lanka, vol. XXXIV. http:// www.cbsl.gov.lk/pics_n_docs/10_pub/_docs/statistics/other/econ _\&_ss_2013_e.pdf (2013). Accessed 10 Jan 2014

30. Sri Lanka Energy Balance: Sustainable Energy Authority. http:// www.info.energy.gov.lk/ (2013). Accessed 28 Nov 2013

31. Ceylon Electricity Board: Colombo. http://www.ceb.lk/sub/pub lications/statistical.aspx (2014). Accessed 11 Jan 2014

32. Ceylon Electricity Board, Transmission and Generation Planning Branch Transmission Division: Long Term Generation Expansion Plan 2013-2032. http://www.pucsl.gov.lk/english/ wp-content/uploads/2013/11/LTGEP\%202013-2032.pdf (2012)

33. Udawatta, L., Perera, A.: Analysis of sensory information for efficient operation of energy management systems in commercial hotels. Department of Electrical Engineering University of Moratuwa, Sri Lanka. Institute of Particle Science \& Engineering University of Leeds, UK. EJSE Special Issue: Wireless Sensor Networks and Practical Applications. http://eprints. whiterose.ac.uk/43282/ (2010)

34. International Finance Corporation, World Bank Group: Ensuring Sustainability in Sri Lanka's Growing Hotel Industry. http:// www.ifc.org/wps/wcm/connect/region_ext_content/regions/sou th+asia/publications/ensuring + sustainability (2013)

35. Household Income and Expenditure Survey Final Report: Sri Lanka Department of Census and Statistics Ministry of Finance and Planning. http://www.statistics.gov.lk/HIES/HIES2009_ 10FinalReportEng.pdf (2011)

36. Sugathapala, T.: Director General Sri Lanka Sustainable Energy Authority, Ministry of Environment and Renewable Energy. Current status and issues of sustainable energy domain in Sri Lanka-Public Powerpoint Presentation (2013)

37. United Nations Development Programme: Sri Lanka global environment facility project document. Promoting sustainable biomass energy production and modern bio-energy technologies. http://www.undp.org/content/dam/srilanka/docs/environment/GE F-Biomass \%20Energy\%20(FSP)\%20PIMS\%204226\%20SRL $\% 20$ Biomass \%20ProDoc\%20draft \%20for $\% 20$ signature $\% 20$ March $\% 20$ 28\%202013.pdf (2013)

38. USAID ECO-III Project International Resources Group 2, Balbir Saxena Marg, Hauz Khas, New Delhi, India: Energy Efficiency in Hospitals Best Practice Guide. http://eco3.org/wp-content/ plugins/downloads-manager/upload/Energy\%20Efficiency $\% 20$ in $\% 20$ Hospitals-\%20For\%20Printing\%20(26\%20Sep\%202011). pdf (2011)

39. Organisation for Economic Co-operation and Development, OECD: Education at a Glance 2013. OECD indicators. http:// www.oecd.org/edu/eag.htm (2013)

40. Sri Lanka Ministry of Health: Hospitals and bed strength in Sri Lanka. http://203.94.76.60/nihs/BEDS/Beds2010/Beds2010.pdf (2010)

41. World Health Organization, WHO: World Health Statistics 2013. http://www.who.int/gho/publications/world_health_statis tics/2013/en/ (2013) 
42. World Health Organization, WHO: Balancing the demand and supply of doctors in Sri Lanka. http://whosrilanka.heal threpository.org/bitstream/123456789/377/1/Balancing\%20the\%2 0demand $\% 20$ and $\% 20$ supply $\% 20$ of $\% 20$ doctors $\% 20 \mathrm{in} \% 20$ Sri $\% 20$ Lanka.pdf (2006)

43. Climate Parliament, Parliamentary Action for Renewable Energy Renewable Energy for India's Human Resource Development: Powering Health Centres under the National Rural Health Mission (NRHM) with clean energy sources. http://www.clima teparl.net/cpcontent/Publications/RE\%20Occassional\%20Paper $\% 20$ No.\%203\%20-\%20SPV\%20for\%20Rural $\% 20$ Health $\% 20$ Cen tres.pdf (2011)

44. US AID India: Energy assessment guide for commercial buildings. http://www.beeindia.in/schemes/documents/ecbc/eco3/ecbc/Energ y\%20Assessment\%20Guide\%20for\%20Buildings.pdf (2010)

45. Private Health Services Regulatory Council: Colombo. http:// www.phsrc.lk/findinstitute.php (2014). Accessed 13 Jan 2014

46. Lee, C.C., Chang, C.P.: Energy consumption and GDP revisited: a panel analysis of developed and developing countries. Energy Econ. 29, 1206-1223 (2007)

47. International Monetary Fund: World Economic and Financial Surveys, World Economic Outlook. http://www.imf.org/exter nal/pubs/ft/weo/2013/02/pdf/text.pdf (2013)

48. Indralal De Silva, W.: A population projection of Sri Lanka for the new millennium 2001-2101: trends and implications. http:// www.ihp.lk/publications/publication.html?id=706 (2007)

49. United Nations Data: Sri Lanka population growth rate \%. http:// data.un.org/Data.aspx?q=sri+lanka\&d=PopDiv\&f=variableID $\%$ 3a47\%3bcrID\%3a144 (2012)

50. Apergis, N., Payne, J.E.: Renewable and non-renewable energy consumption-growth nexus: evidence from a panel error correction model. Energy Econ. 34, 733-738 (2012)

51. Sri Lanka Energy Managers Association: SLEMA J., 16 (2013)

52. Samaratunga, R.H.S.: Secretary, Ministry of Petroleum Industries. Challenges of the Sri Lanka's Petroleum Industry (2012)

53. Sri Lanka Sustainable Energy Authority: Colombo. http://www. energy.gov.lk/sub_pgs/energy_renewable_hydro_potential.html (2014). Accessed 20 Feb 2014

54. Deheragoda, C.K.M.: Chairman Sri Lanka Sustainable Energy Authority. Renewable energy development in Sri Lanka. http:// www.techmonitor.net/tm/images/e/ee/09nov_dec_sf6.pdf (2009). Accessed 05 Nov 2013

55. Ceylon Electricity Board: Present status of non-conventional renewable energy sector (as of 31/12/2013). http://www.ceb.lk/ sub/db/op_presentstatus.html (2013). Accessed 10 Nov 2013

56. Public Utilities Commission of Sri Lanka: Off-Grid Electrification using Micro hydro power schemes, Sri Lankan Experience. A survey and Study on existing off-grid electrification schemes. http://www.pucsl.gov.lk/english/wp-content/uploads/ 2013/03/Off-Grid-5-2name.pdf (2012). Accessed 15 Nov 2013

57. Greenpeace: Global wind energy outlook. http://www.gwec.net/ wp-content/uploads/2012/11/GWEO_2012_lowRes.pdf (2012)

58. United Nations Framework Convention on Climate Change (UNFCCC): Project design document form for Clean Development Mechanism (CDM) project activities, version $04.1 \mathrm{http}: / /$ cdm.unfecc.int/filestorage/F/A/7/FA7P6OTR9NGX48Y1LKCH 3QU0VZEJ2S/9675\%20PDD.pdf?t=dGd8bjFkNGh3fDB62_Ys RPLP-Nlq1Y6hKxLD (1997)

59. De Silva TSP: Develop strategies to increase NCRE power generation in Sri Lanka above $10 \%$ level by the year 2015 (2012)

60. Perera, K.K.C.K., Rathnasiri, P.G., Sugathapala, A.G.T.: Sustainable biomass production for energy in Sri Lanka. Biomass Bioenergy 25, 541-556 (2003)

61. Ministry of Power and Energy: National Energy Policy and Strategies of Sri Lanka (2008)
62. Wickramasinghe, A.: (Practical Action). Sri Lanka: integrating biofuels into small farm operations for income and economic development (2009)

63. Munasinghe, S.: Biogas technology and integrated development (experiences from Sri Lanka). In: World Energy Congress VI (WREC 2000). Elsevier (2000)

64. Bhattacharyya, S.C.: Energy access programmes and sustainable development: a critical review and analysis. Energy Sustain. Dev. 16, 260-271 (2012)

65. Sri Lanka Renewable Energy Report: Asian and Pacific Centre for Transfer of Technology of the United Nations-Economic and Social Commission for Asia and the Pacific (ESCAP) (2009)

66. Wijayatunga, P.D.C.: Socio-economic impact of solar home systems in rural Sri Lanka: a case-study. Energy for Sustainable Development 1, vol. IX, no. 2 (2005)

67. McEachern, M., Hanson, S.: Socio-geographic perception in the diffusion of innovation: solar energy technology in Sri Lanka. Energy Policy 36, 2578-2590 (2008)

68. Werner, C.H., Breyer, C.H., Gerlach, A.: Analysis and forecast of global installed photovoltaic capacity and identification of hidden growth markets. In: 28th European Photovoltaic Solar Energy Conference. Paris, France (2013)

69. Mangala, P.S., Wijetilake, S.: The potential of geothermal energy resources in Sri Lanka. United Nations University, Geothermal Training Programme. Report 34 (2011)

70. Grid Interconnection Mechanisms for Off-Grid Electricity Schemes in Sri Lanka: Public Utilities Commission of Sri Lanka. http://www.pucsl.gov.lk/english/wp-content/uploads/ 2013/06/Grid-Interconnection-mechanisms-for-off-grid-electrici ty-schemes.pdf (2013). Accessed 1 Dec 2013

71. Miller, D., Hope, C.: Learning to lend for off-grid solar power: policy lessons from World Bank loans to India, Indonesia, and Sri Lanka. Energy Convers. Manag. 47, 1179-1191 (2006)

72. Palit, D., Chaurey, A.: Off-grid rural electrification experiences from South Asia: Status and best practices. Energy Sustain. Dev. 15, 266-276 (2011)

73. Subhes, C., Bhattacharyya, N.: Financing energy access and offgrid electrification: A review of status, options and challenges. Renew. Sustain. Energy Rev. 20, 462-472 (2013)

74. United Nations: The Millennium Development Goals Report. http://www.un.org/millenniumgoals/pdf/report-2013/mdg-report -2013-english.pdf (2013). Accessed 3 Nov 2013

75. REN21: Renewables 2013 Global Status Report. Washington DC. http://www.ren21.net/ren21activities/globalstatusreport. aspx (2013). Accessed 10 Dec 2013

76. Dhanapala K, Wijayatunga P. Economic and environmental impact of micro-hydro and biomass based electricity generation in the Sri Lanka tea plantation sector. Energy for Sustainable Development 1 Volume VI No. 11 March 2002

77. Morimoto, R., Hope, C.: The impact of electricity supply on economic growth in Sri Lanka. Energy Econ. 26, 77-85 (2004)

78. Priyantha, D.C.: Wijayatunga, Jayalath MS. Assessment of economic impact of electricity supply interruptions in the Sri Lanka industrial sector. Energy Convers. Manag. 45, 235-247 (2004)

79. The World Bank: Country indicators. http://data.worldbank.org/ indicator/NY.GDP.MKTP.CD/countries/LK?display=graph (2014). Accessed on 20 Jan 2014

80. World Health Organization (WHO): Health in the Green Economy, Co-benefits to health of climate change mitigationhousing sector. http://www.who.int/hia/hgehousing.pdf (2011). Accessed 10 Dec 2013

81. Moretti, E., Bonamente, E., Buratti, C., Cotana, F.: Development of innovative heating and cooling systems using renewable energy sources for non-residential buildings. Energies 6(10), 5114-5129 (2013) 
82. Gallo, D., Landi, C., Luiso, M., Morello, R.: Optimization of experimental model parameter identification for energy storage systems. Energies 6(9), 4572-4590 (2013)

83. Gómez-Gil, F.J., Wang, X., Barnett, A.: Analysis and prediction of energy production in concentrating photovoltaic (CPV) installations. Energies 5(3), 770-789 (2012)

84. Wu, Y., Yang, W., Blasiak, W.: Energy and exergy analysis of high temperature agent gasification of biomass. Energies 7(4), 2107-2122 (2014)

85. Sri Lanka Energy Balance: Sustainable energy authority. http:// www.info.energy.gov.lk/ (2013). Accessed 28 Nov 2013

86. Ceylon Electricity Board: Colombo. http://www.ceb.lk/sub/pub lications/statistical.aspx (2014). Accessed 11 Jan 2014

87. Statistics: FAQ. International Energy Agency. http://www.iea. org/statistics/resources/questionnaires/faq/ (2014). Accessed 10 Jan 2014

88. Sri Lanka Ministry of Power and Energy: Generation plants. Colombo. http://powermin.gov.lk/english/?page_id=1507 (2014). Accessed 03 Jan 2014

89. Public Utilities Commission of Sri Lanka: Generation performance in Sri Lanka 2012. http://www.pucsl.gov.lk/tamil/wpcontent/uploads/2013/07/Gen\%20Performance_2012.pdf (2013)

90. Public Utilities Commission of Sri Lanka: Achievements of renewable energy targets in Sri Lanka 2011. http://www.pucsl. gov.lk/tamil/wp-content/themes/pucsl/pdfs/annual_report_achie vements_of_renewable_energy_targets_2011.pdf (2012)

91. Ceylon Electricity Board, Transmission and Generation Planning Branch Transmission Division: Long term generation expansion plan 2013-2032. http://www.pucsl.gov.lk/english/wpcontent/uploads/2013/11/LTGEP\%202013-2032.pdf (2012)

92. Grid Interconnection Mechanisms for Off-Grid Electricity Schemes in Sri Lanka: Public Utilities Commission of Sri Lanka. http://www.pucsl.gov.lk/english/wp-content/uploads/ 2013/06/Grid-Interconnection-mechanisms-for-off-grid-electrici ty-schemes.pdf (2013). Accessed 1 Dec 2013

93. Udawatta, L., Perera, A.: Analysis of sensory information for efficient operation of energy management systems in commercial hotels. Department of Electrical Engineering University of Moratuwa, Sri Lanka. Institute of Particle Science \& Engineering University of Leeds, UK. EJSE Special Issue: Wireless Sensor Networks and Practical Applications. http://eprints. whiterose.ac.uk/43282/ (2010)

94. International Finance Corporation, World Bank Group: Ensuring Sustainability in Sri Lanka's Growing Hotel Industry. http:// www.ifc.org/wps/wcm/connect/region_ext_content/regions/sou th+asia/publications/ensuring+sustainability (2013)

95. Household Income and Expenditure Survey Final Report: Sri Lanka Department of Census and Statistics Ministry of Finance and Planning. http://www.statistics.gov.lk/HIES/HIES2009 10FinalReportEng.pdf (2011)

96. Sugathapala, T.: Director General Sri Lanka Sustainable Energy Authority, Ministry of Environment and Renewable Energy. Current status and issues of sustainable energy domain in Sri Lanka-Public Powerpoint Presentation (2013)

97. United Nations Development Programme: Sri Lanka global environment facility project document. Promoting sustainable biomass energy production and modern bio-energy technologies. http://www.undp.org/content/dam/srilanka/docs/environment/GE F-Biomass\%20Energy\%20(FSP)\%20PIMS\%204226\%20SRL\%2 0Biomass\%20ProDoc\%20draft\%20for\%20signature\%20March $\% 2$ 028\%202013.pdf (2013)

98. Sri Lanka Sustainable Energy Authority: Colombo. http://www. energy.gov.lk/sub_pgs/energy_renewable_hydro_potential.html. Accessed 20 Feb 2014

99. Deheragoda, C.K.M.: Chairman Sri Lanka Sustainable Energy Authority. Renewable energy development in Sri Lanka. http:// www.techmonitor.net/tm/images/e/ee/09nov_dec_sf6.pdf (2009). Accessed 05 Nov 2013

100. Ceylon Electricity Board: Present Status of Non-Conventional Renewable Energy Sector (as of 31/12/2013). http://www.ceb. lk/sub/db/op_presentstatus.html (2013). Accessed 10 Nov 2013

101. Greenpeace: Global wind energy outlook. http://www.gwec.net/ wp-content/uploads/2012/11/GWEO_2012_lowRes.pdf (2012)

102. Ministry of Power and Energy: National Energy Policy and Strategies of Sri Lanka (2008)

103. Munasinghe, S.: Biogas technology and integrated development (experiences from Sri Lanka). World Energy Congress VI (WREC2000). Elsevier (2000)

104. European Commission Energy Forum: Incorporating social and environmental concerns in long term electricity generation expansion planning in Sri Lanka. http://www.efsl.lk/reports/WADE\%20Gen eration\%20Planning\%20study\%20report.pdf (2006). Accessed 10 Dec 2013

105. United Nations Development Program: Sri Lanka global environment facility project document (2013)

106. World Health Organization, WHO: Fuel for Life, household energy and health. http://www.who.int/indoorair/publications/ fuelforlife/en/ (2006). Accessed 05 Dec 2013

107. Millo, F., Rolando, L., Fuso, R.: Real world operation of a complex plug-in hybrid electric vehicle: analysis of its $\mathrm{CO}_{2}$ emissions and operating costs. Energies 7(7), 4554-4570 (2014)

108. Nuesch, T., Cerofolini, A., Mancini, G., Cavina, N., Onder, C., Guzzella, L.: Equivalent consumption minimization strategy for the control of real driving NOx emissions of a diesel hybrid electric vehicle. Energies 7(5), 3148-3178 (2014)

109. Priyantha, D., Wijayatunga, D.C., Fernando, W.J.L.S., Shrestha, R.M., Siriwardena, K., Attalage, R.A.: Economy wide emission impacts of carbon and energy tax in electricity supply industry: a case study on Sri Lanka. Energy Convers. Manag. 48, 1975-1982 (2007)

110. Priyantha, D., Wijayatunga, D.C., Fernando, W.J.L.S., Shrestha, R.M., Siriwardena, K., Attalage, R.A.: Strategies to overcome barriers for cleaner generation technologies in small developing power systems: Sri Lanka case study. Energy Convers. Manag. 47, 1179-1191 (2006)

111. Priyantha, D., Wijayatunga, D.C., Fernando, W.J.L.S., Shrestha, R.M.: Impact of distributed and independent power generation on greenhouse gas emissions: Sri Lanka. Energy Convers. Manag. 45, 3193-3206 (2004)

112. World Nuclear Association: Comparison of Lifecycle Greenhouse Gas Emissions of Various Electricity Generation Sources. http:// www.world-nuclear.org/uploadedFiles/org/WNA/Publications/Wo rking_Group_Reports/comparison_of_lifecycle.pdf (2011). Accessed 01 March 2014

113. Intergovernmental Panel on Climate Change, IPCC: Climate Change 2013, the physical science basis. https://www.ipcc.ch/ report/ar5/wg1/ (2013). Accessed 02 March 2014

114. International Energy Agency: $\mathrm{CO}_{2}$ emissions from fuel combustion. http://www.iea.org/publications/freepublications/pub lication/name,43840, en.html (2013). Accessed 02 March 2014

115. US Energy Information Administration, EIA: Voluntary Reporting of Greenhouse Gases Program, Fuel Emission Coefficients. http://www.eia.gov/oiaf/1605/coefficients.html. Accessed 02 March 2014

116. US Department of Energy. National Renewable Energy Laboratory, NREL: Wind LCA Harmonization. http://www.nrel. gov/docs/fy13osti/57131.pdf. Accessed 10 Dec 2013

117. US Department of Energy: National Renewable Energy Laboratory, NREL. Life Cycle Greenhouse Gas Emissions from Solar Photovoltaics. http://www.nrel.gov/docs/fy13osti/56487. pdf. Accessed 05 Jan 2014 
118. Masanet, E., Chang, Y., Gopal, A.R., Larsen, P., Morrow, W.R., Sathre, R., Shehabi, A., Zhai, P.: Life-Cycle Assessment of Electric Power Systems (2013)

119. Houshfar, E., Lovas, T., Skreiberg, O.: Experimental investigation on NOx reduction by primary measures in biomass combustion: straw, peat, sewage sludge. Forest residues wood pellets. Energies 5, 270-290 (2012)

120. Moradian, F., Pettersson, A., Svard, S.H., Richards, T.: Cocombustion of animal waste in a commercial waste-to-energy BFB boiler. Energies 6, 6170-6187 (2013)

121. Tchapda, A.H., Pisupati, S.V.: A review of thermal co-conversion of coal and biomass/waste. Energies 7, 1098-1148 (2014)

122. IEA, 2011, Biofuels for Transport: IEA Technology Roadmap, IEA-OECD. Paris. http://www.iea.org/roadmaps (2011)

123. IEA, 2010, Energy Technology Perspectives 2010, IEA-OECD. Paris. http://www.iea.org (2010)

124. IEA-ETSAP and International Renewable Energy Agency, IRENA: Production of Liquid Biofuels, Technology Brief. http://www.irena.org/Publications/ (2013)

125. Chum, H., Faaij, A., Moreira, J., Berndes, G., Dhamija, P., Dong, H., Gabrielle, B., Goss, A., Lucht, W., Mapako, M., Masera, O., McIntyre, T., Minowa, T., Pingoud, T.: Bioenergy. In: IPCC Special Report on Renewable Energy Sources and Climate Change Mitigation. Cambridge University Press, Cambridge, United Kingdom and New York (2011)

126. World Health Organization (WHO): Sri Lanka Cooperation Strategy at a glance. http://www.who.int/countryfocus/coopera tion_strategy/ccsbrief_lka_en.pdf (2013). Accessed 13 Dec 2013

127. United Nations Educational, Scientific and Cultural Organization (UNESCO): Education profile, Sri Lanka. http://stats.uis. unesco.org/unesco/TableViewer/document.aspx? ReportId= $121 \& I F \_L a n g u a g e=e n g \& B R \_C o u n t r y=7340 \& B R \_$Region $=$ 405352013. Accessed 1 Dec 2013

128. Organisation for Economic Co-operation and Development, OECD: Education at a Glance 2013. OECD indicators. http:// www.oecd.org/edu/eag.htm (2013)

129. Sri Lanka Ministry of Health: Hospitals and bed strength in Sri Lanka. http://203.94.76.60/nihs/BEDS/Beds2010/Beds2010.pdf (2010)

130. Public Utilities Commission of Sri Lanka: Off-Grid Electrification using micro hydro power schemes, Sri Lankan
Experience. A survey and Study on existing off-grid electrification schemes. http://www.pucsl.gov.lk/english/wp-content/ uploads/2013/03/Off-Grid-5-2name.pdf (2012). Accessed 15 Nov 2013

131. Wickramasinghe, A. (Practical Action) 2009. Sri Lanka: Integrating Biofuels into Small Farm Operations for Income and Economic Development

132. Bhattacharyya, S.C.: Energy access programmes and sustainable development: A critical review and analysis. Energy Sustain. Dev. 16, 260-271 (2012)

133. Sri Lanka Renewable Energy Report: Asian and Pacific Centre for Transfer of Technology of the United Nations-Economic and Social Commission for Asia and the Pacific (ESCAP) (2009)

134. Wijayatunga, PDC: Socio-economic impact of solar home systems in rural Sri Lanka: a case-study. Energy for sustainable development 1, vol. IX, no. 2 (2005)

135. Subhes, C., Bhattacharyya, N.: Financing energy access and offgrid electrification: a review of status, options and challenges. Renew. Sustain. Energy Rev. 20, 462-472 (2013)

136. REN21: Renewables 2013 Global Status Report. Washington DC. http://www.ren21.net/ren21activities/globalstatusreport. aspx (2013). Accessed 10 Dec 2013

137. International Renewable Energy Agency, IRENA: Renewable Power Generation Costs in 2012: an overview. https://www.irena. org/menu/index.aspx $? \mathrm{mnu}=$ Subcat $\&$ PriMenuID $=36 \&$ CatID $=141 \&$ SubcatID=277 (2013). Accessed 27 Dec 2013

138. International Renewable Energy Agency, IRENA: Renewable Energy Technologies: cost analysis series, vol. 1: Power Sector Issue 15/5. Biomass for Power Generation.http://www.irena.org/ DocumentDownloads/Publications/RE_Technologies_Cost_Ana lysis-BIOMASS.pdf (2012). Accessed 15 Feb 2014

139. Dall'O, G., Norese, M.F., Galante, A., Novello, C.: A multicriteria methodology to support public administration decision making concerning sustainable energy action plans. Energies, 6(8), 4308-4330.137 (2013)

140. Kang, H.Y., Hung, M.C., Pearn, W.L., Lee, A.H.I., Kang, M.S.: An integrated multi-criteria decision making model for evaluating wind farm performance. Energies 4(11), 2002-2026 (2011) 